\title{
Contribution à l'inventaire faunistique des Acridiens de l'Archipel du
} Cap-Vert [Orth.]

Jean-François Duranton, Michel Launois, My Hang Launois-Luong, Michel Lecoq

Citer ce document / Cite this document :

Duranton Jean-François, Launois Michel, Launois-Luong My Hang, Lecoq Michel. Contribution à l'inventaire faunistique des Acridiens de l'Archipel du Cap-Vert [Orth.]. In: Bulletin de la Société entomologique de France, volume 88 (3-4), Mars-avril 1983. Livre du Cent Cinquantenaire. Premier congrès international des entomologistes d'expression française. Paris, 6-9 juillet 1982. Comptes rendus des travaux. II. pp. 197-224;

https://www.persee.fr/doc/bsef_0037-928x_1983_num_88_3_18303

Fichier pdf généré le 02/09/2019 


\title{
Contribution à l'inventaire faunistique des Acridiens de l'Archipel du Cap-Vert
}

\author{
[ORTH.] \\ par Jean-François Duranton, Michel Launois, My Hanh Launors-Luong \\ \& Michel LEcoo
}

Si, d'une manière génćrale, la faune acridienne des Iles Macaronésiennes - et, en particulier, des Canaries - est bien connue et a fait l'objet de nombreux travaux, celle des Iles du Cap-Vert a été assez peu prospectée. Les premières signalisations concernant les Acridiens de cette région remontent à la fin du siècle dernier. Les principales sont rapportées dans l'inventaire des Orthoptères africains du Muséum de Lisbonne réalisé par Bolivar (1889). Onze espèces sont alors dénombrées de l'Archipel. Par la suite, Burr (1927) fait une brève relation d'une escale de quelques heures dans les Iles au cours de laquelle il lui fut possible d'identifier approximativement 6 espèces. Il faut ensuite attendre l'analyse faite par Chopard (1936) du matériel récolté en 1934 par Chevalier pour disposer d'un premier inventaire assez complet de la faune acridienne de l'Archipel. Chopard dénombre alors 15 espèces dont 4 seulement déjà signalées par Bolivar, portant ainsi à 22 la liste des espèces d'Acridiens du Cap-Vert. Cette liste sera ensuite essentiellement complétée par Chopard, en 1958, grâce à un important matériel collecté par Lindberg et Panelius au cours de l'hiver 1953-1954. Dix espèces supplémentaires sont alors signalées. Saraiva (1961), dans une compilation bibliographique sur la faune de l'Archipel, répertorie, pour les Acridiens, 30 espèces. Une signalisation nouvelle d'Anacridium est ensuite effectuée par Johnsen (1977). Tout dernièrement (décembre 1978 - janvier 1979) une mission allemande (MM. Bauer, Friebe et Groh) a pu récolter dans les Iles un certain nombre de spécimens d'Orthoptères qui ont été déterminés par Harz (1982). Toutes les espèces mentionnées avaient déjà été signalées de l'Archipel.

Dans l'ensemble, seuls les imagos ont fait l'objet de signalisations. Les larves n'ont jamais reçu de mentions particulières à l'exception de celles d'Oedaleus senegalensis, espèce abondante dans les Iles du Cap-Vert et dont la biologie a été étudiée par Saraiva (1962).

Depuis quelques années, nous avons eu l'occasion d'effectuer plusieurs missions d'assistance au Service de la Protection des Végétaux des Iles du Cap-Vert dans le cadre de la lutte contre les Acridiens ravageurs. A ces occasions, un inventaire systématique de la faune acridienne a été entrepris dans sept des îles principales de l'Archipel (92\% des terres émergées de ce pays). Cet inventaire - destiné à nous permettre de préciser, entre autres, le tempérament écologique des diverses espèces - a porté également sur la composition floristique du tapis végétal ainsi que sur de nombreuses autres composantes de l'environnement acridien.

En prélude aux études écologiques, il convient de procéder ici à une analyse critique des signalisations (les nôtres et celles des auteurs antérieurs) afin d'établir un inventaire actualisé de l'acridofaune de cet Archipel, le plus méridional de la Macaronésie. Les principales caractéristiques des peuplements acridiens seront dégagées; elles ćclaireront les affinités biogéographiques de cette faune insulaire après que le milieu et les méthodes d'investigation aient été brièvement décrits. 


\section{Le milieu.}

L'Archipel du Cap-Vert fait classiquement partie - avec les Açores, Madère, l'Archipel des Salvages et les Canaries - des Iles Macaronésiennes. Cet Archipel est constitué par un ensemble de 14 îles, dont 9 îles principales et 5 îlots inhabités, d'origine essentiellement volcanique; l'émersion s'est vraisemblablement produite au milieu du tertiaire. La superficie totale de l'Archipel est de l'ordre de $4033 \mathrm{~km}^{2}$; il est situé dans l'Atlantique Nord à environ $500 \mathrm{~km}$ à l'Ouest de Dakar.

Deux groupes d'îles peuvent être distingués - les îles basses situées à l'Est: Maio, Boa-Vista, Sal, au relief plat, culminant au maximum à $400 \mathrm{~m}$ - et les îles hautes, plus à l'Ouest, au relief très accidenté et dont les sommets sont fréquemment noyés dans les nuages: San Antao, San Vicente, les îlots de Santa Luzia, Branco et Razo, puis San Nicolau, Santiago, Fogo (point culminant de l'Archipel avec $2835 \mathrm{~m}$ d'altitude), Brava et enfin les îlots Seccos ou Rombo.

Ces îles sont, la plus grande partie de l'année, sous l'influence des alizés de Nord-Est qui provoquent, sur les îles hautes, la formation d'une barrière nuageuse sur tous les versants Nord-Est exposés aux vents, déterminant ainsi l'un des principaux contrastes écologiques des îles : celui existant entre les versants Sud-Ouest, arides, et les versants Nord-Est, beaucoup plus humides. Le deuxième facteur écologique fondamental de différenciation des biotopes acridiens est évidemment l'altitude, les zones basses étant beaucoup plus xérotrophes que les sommets.

Les pluies sont essentiellement des pluies de mousson survenant de juillet à octobre, en faible quantité. La caractéristique essentielle des précipitations réside dans leur irrégularité d'une année à l'autre (des sécheresses complètes de plusieurs années ont été observées) et pour une même année entre les diverses îles. Dans l'ensemble, le climat est de type sahélien, aride à semi-aride, avec des températures modérées par les influences océaniques et l'altitude.

\section{Méthodes d'étude.}

Nous avons pu effectuer dans l'Archipel trois missions, d'environ un mois chacune, pendant trois années consécutives. Ces missions ont été réalisées en fin de saison des pluies, époque qui jusqu'à présent n'avait guère fait l'objet d'investigations et qui permettait surtout de réunir un maximum de chances de trouver la plus grande diversité d'espèces, aussi bien les Acridiens passant la longue saison sèche en diapause imaginale que ceux, à diapause embryonnaire, dont les adultes et les larves ne sont guère présents que pendant la saison des pluies.

L'île de Santiago a ainsi été prospectée du 27 septembre au 1 $1^{\text {er }}$ novembre 1979 , les îles de San Nicolau, Boa-Vista et Maio du 9 octobre au 13 novembre 1980 et enfin les îles de San Antao, San Vicente et Fogo du 14 septembre au $1^{\text {er }}$ novembre 1981.

Pour l'ensemble de ces 3 années, 621 relevés ont été effectués. Les différents points de relevés ont été choisis selon une procédure d'échantillonnage stratifié tenant compte à la fois de la diversité écologique des biotopes en présence et de la superficie globale de chaque île. Pour certaines d'entres elles, mal desservies par le réseau de routes et de pistes muletières (San Antao en particulier), des parties importantes ont échappé aux prospections par manque de temps. L'échantillonnage réalisé assure cependant une bonne couverture géographique des Iles mais l'on ne peut exclure que certains biotopes aient pu passer inaperçus (parois rocheu- 
ses ou milieux hygrotrophes en particulier, dont certains n'ont été découverts qu'après de longues marches hors des pistes balisées).

Chaque relevé est effectué au sein d'une surface floristiquement (donc écologiquement) homogène, durant environ 30 à 40 minutes. Une évaluation numérique globale de la population acridienne (larves et ailés) est réalisée par des séries de comptages à vue sur des surfaces de $1 \mathrm{~m}^{2}$. Un échantillonnage des différentes espèces est effectué en combinant captures au filet et déterminations à vue pour les espèces les plus communes. Une description détaillée de la méthodologie utilisée pourra ctre trouvée par ailleurs (Launois 1972, Lecoq 1978, Duranton et al. 1982) (').

\section{Inventaire de la faune acridienne des Iles du Cap-Vert.}

L'inventaire critique des différentes espèces acridiennes signalées à ce jour de l'Archipel est fourni en annexe. Pour notre part, sur l'ensemble des 621 relevés et grâce à l'examen de plusieurs dizaines de milliers d'individus (75000 environ), nous avons pu dénombrer 23 espèces ce qui, compte tenu des travaux antérieurs, porte à 35 le nombre total d'Acridiens signalés des Iles du Cap-Vert (tabl. I). L'Archipel n'ayant pas, ou exceptionnellement, été prospecté en fin de saison des pluies, la quasi totalité de nos signalisations sont nouvelles pour les mois de septembre et octobre (tabl. II).

Ce chiffre de 35 espèces est évidemment faible si on le compare aux quelque 200 espèces d'Acridions des zones soudanienne et sahélienne d'Afrique de l'Ouest (Launois 1978, Lecoq $1980 \mathrm{a}$ ). Cet appauvrissement est en fait une caractéristique générale des faunes insulaires. Par ailleurs, cet effectif correspond à un maximum qui ne tient compte ni des quelques problèmes de taxonomie restant à résoudre (quelques synonymies possibles, en particulier), ni du fait que la présence de nombreuses espèces paraît tout à fait accidentelle dans l'Archipel.

On remarquera que 6 espèces n'ont été signalées qu'une seule fois des Iles du Cap-Vert, au siècle dernier, et n'ont pas été retrouvées depuis. Il s'agit d'une part, de Anacridium wernerellum signalé par Dirsh \& Uvarov (1953) d'après un exemplaire capturé par Delaunay en 1882 - exemplaire conservé au Muséum de Paris mais que nous n'avons pu retrouver - et, d'autre part, de Phymateus aegrotus, Anacridium aegyptium, Cyrtacanthacris $t$. tatarica, Acrida turrita et Truxalis nasuta signalés par Bolivar (1889) d'après des exemplaires capturés par Ferreira Borges et Custodio Duarte conservés au Muséum de Lisbonne. On ne manquera pas, à ce propos, de relever que ces 5 dernières espèces constituent la moitié des Acridiens signalés par Bolivar des Iles du Cap-Vert alors que de nombreuses espèces beaucoup plus abondantes ne figurent pas dans sa liste. Dans l'ensemble, il ne peut s'agir, au mieux, que d'espèces tout à fait accidentelles à l'égal, d'ailleurs, de Epacromius tergestinus, espèce paléarctique dont un individu a été signalé dans l'Archipel par Chopard (1958 b), d'Ornithacris turbida cavroisi, signalé par Uvarov (1924) d'après un exemplaire, conservé au Muséum de Paris, en provenance de San Vicente, d'Anacridium eximium dont un mâle a été décrit par Johnsen (1977) et de Brachycrotaphus trvxalicerus dont un mâle également a été capturé au cours de l'expédition Lindberg en 1954 sur Santiago (Chopard 1958 b).

(1) Une description détaillée de l'environnement assortie d'un relevé floristique selon la méthode phytosociologique stigmatiste est également réalisée dans chaque site prospecté. Ces données seront ultérieurement utilisées pour caractériser les biotopes types de chaque espèce. 
Par ailleurs, certaines espèces ont un statut taxonomique incertain et les problèmes qui demeurent seront évoqués, dans l'annexe, au niveau de chaque espèce concernée. Mentionnons cependant ici deux exemples, dont le cas du genre Pyrgomorpha pour lequel le problème se pose de savoir s'il s'agit, dans les Iles, de $P$. conica tereticornis, décrit par Brullé en 1840 - auquel cas il s'agirait d'une sous-

Paratettix meridionalis
Pyrgomorpha cognata
Pyrgomorpha conica tereticornis
Catantops axillaris axillaris
Schistocerca gregaria
Anacridium wernerellum
Anacridium m. melanorhodon
Anacridium eximium
Nomadacris septemfasciata insularis
Ornithacris turbida cavroisi
Heteracris littoralis
Euprepocprifas insularis
Acorypha clara
Pseudosphingonotus savignyi
Sphingonotus c. canariensis
Sphingonotus r. rubescens
Sphingonotus rubescens burri
Aiolopus thalassinus
Aiolopus strepens
Aiolopus s. simulatrix
Epacromius tergestinus
Trilophidia conturbata
Acrotylus longipes
Acrotylus patruelis
Oedaleus s. senegalensis
Oedaleus senegalensis dimidiatus
Locusta migratoria
Leva bonneti
Leva epacromioides
Ochrilidia geniculata
Brachycrotaphus tryxalicerus

\begin{tabular}{|c|c|c|c|c|c|c|c|c|c|}
\hline A & V & SL & II & $S$ & BV & M & $\mathrm{T}$ & F & BR \\
\hline $\mathrm{x}$ & + & - & + & • & + & - & $\mathrm{X}$ & . & + \\
\hline • & • & . & - & . & - & - & - & - & - \\
\hline+ & + & . & + & + & + & + & + & + & \\
\hline $\mathrm{x}$ & $\mathrm{X}$ & + & $\mathrm{x}$ & + & $\mathrm{X}$ & $\mathrm{x}$ & $\mathrm{x}$ & $\mathrm{x}$ & + \\
\hline$\bullet$ & $x$ & + & $\bullet$ & + & $\mathrm{x}$ & $\bullet$ & + & . & . \\
\hline$\therefore$ & + & - & - & - & - & - & . & - & . \\
\hline $\mathrm{x}$ & $\mathrm{x}$ & . & $x$ & . & $x$ & $x$ & $\mathrm{X}$ & $\mathrm{x}$ & . \\
\hline • & + & . & - & • & • & . & . & . & . \\
\hline+ & - & . & • & . & . & . & + & $\mathrm{X}$ & + \\
\hline$\therefore$ & + & • & $\dot{0}$ & • & $\therefore$ & - & $\dot{0}$ & - & . \\
\hline $\mathrm{x}$ & $X$ & . & $\mathrm{X}$ & + & $x$ & $x$ & • & - & . \\
\hline • & i & • & - & $\dot{2}$ & $\dot{ }$ & : & $\dot{\mathrm{x}}$ & $\dot{\mathrm{x}}$ & · \\
\hline - & + & • & + & $?$ & + & + & $\mathrm{X}$ & X & . \\
\hline$\therefore$ & - & - & - & . & • & - & $\mathrm{X}$ & • & . \\
\hline $\mathrm{X}$ & $\mathrm{X}$ & • & $\mathrm{X}$ & + & $\mathrm{X}$ & $\mathrm{X}$ & $\mathrm{X}$ & $\mathrm{X}$ & . \\
\hline $\mathrm{x}$ & $\mathrm{X}$ & + & $\mathrm{X}$ & . & $\mathrm{X}$ & - & $\bullet$ & $\bullet$ & . \\
\hline $\mathrm{X}$ & + & $?$ & $\mathrm{X}$ & + & • & . & ? & + & ? \\
\hline $\mathrm{x}$ & $\mathrm{X}$ & . & + & - & + & - & $\mathrm{X}$ & $\mathrm{x}$ & + \\
\hline+ & + & . & - & • & • & $\dot{ }$ & + & + & . \\
\hline - & - & & • & • & - & - & - & - & . \\
\hline+ & - & • & - & • & • & $\therefore$ & $\therefore$ & $\therefore$ & $\therefore$ \\
\hline . & - & • & - & • & - & $x$ & $\mathrm{X}$ & $\mathrm{X}$ & $x$ \\
\hline X & $x$ & . & $x$ & + & $x$ & $x$ & $x$ & $\mathrm{x}$ & + \\
\hline $\mathrm{X}$ & $\mathrm{X}$ & . & $\mathrm{X}$ & • & $\mathrm{X}$ & $x$ & $\mathrm{X}$ & $\mathrm{X}$ & . \\
\hline $\mathrm{X}$ & $X$ & . & $x$ & + & $\mathrm{x}$ & $\mathrm{X}$ & $\mathrm{X}$ & $\mathrm{x}$ & + \\
\hline$\bullet$ & • & • & ○ & • & • & • & • & - & . \\
\hline $\mathrm{X}$ & - & . & + & . & . & . & - & $\mathrm{X}$ & + \\
\hline$\bullet$ & • & • & ㄴ & . & + & - & $\mathrm{X}$ & $\mathrm{X}$ & . \\
\hline & • & " & 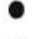 & • & $\mathrm{x}$ & 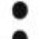 & $\bullet$ & $\bullet$ & . \\
\hline$\cdot$ & $\cdot$ & $\cdot$ & $\cdot$ & $\cdot$ & $\mathrm{X}$ & • & : & - & . \\
\hline - & $\cdot$ & $\cdot$ & $\cdot$ & $\cdot$ & $\cdot$ & $\cdot$ & + & - & $\cdot$ \\
\hline
\end{tabular}

Espèces signalées de l'Archipel sans localisation:

Phymateus aegrotus

Anacridium aegyptium

Cyrtacanthacris $t$. tatarica

Acrida turrita

Truxalis nasuta

Tableau I. Liste des Acridiens signales de l'Archipel du Cap-Vert.

A, San Antao; V, San Vicente; SL, Santa Luzio; N, San Nicolau;

S, Sal; BV, Boa Vista; M, Maio; T, Santiago; F, Fogo; BR, Brava;

R, Ilots Rombo.

. espèce non signalé; + , espèce signalée non retrouvee; $x$,

signalisation confirmé; 0 , signalisation nouvelle; $?$, signalisation

douteuse.

espèce à affinités méditerranéennes - ou bien s'il ne s'agit pas plutôt du $P$. cognata largement distribué en Afrique de l'Ouest à des latitudes comparables. Seule une révision complète du genre pourrait ici clarifier le problème.

Rappelons aussi le cas du genre Aiolopus. Si nous avons bien retrouvé $A$. thalassinus, nous n'avons pu capturer aucun $A$. strepens, signalé à deux reprises par Chopard (1958 b) et Harz (1982) mais, par contre, nous avons capturé en assez grande abondance $A$. $s$. simulator et le problème subsiste d'une confusion possible 
par les auteurs antérieurs de ces deux espèces, méditerranéenne pour la première, de zone soudano-sahélienne pour la seconde qu'il semblerait d'ailleurs beaucoup plus logique de rencontrer dans l'Archipel. Cette confusion a effectivement été vérifiée dans un cas (cf. annexe).

Compte tenu de toutes ces remarques, l'acridofaune des Iles du Cap-Vert se réduit à un ensemble de 23 espèces se reproduisant régulièrement sur les îles, c'est-à-dire les 23 espèces (ou sous-espèces) observées au cours de nos 3 missions.

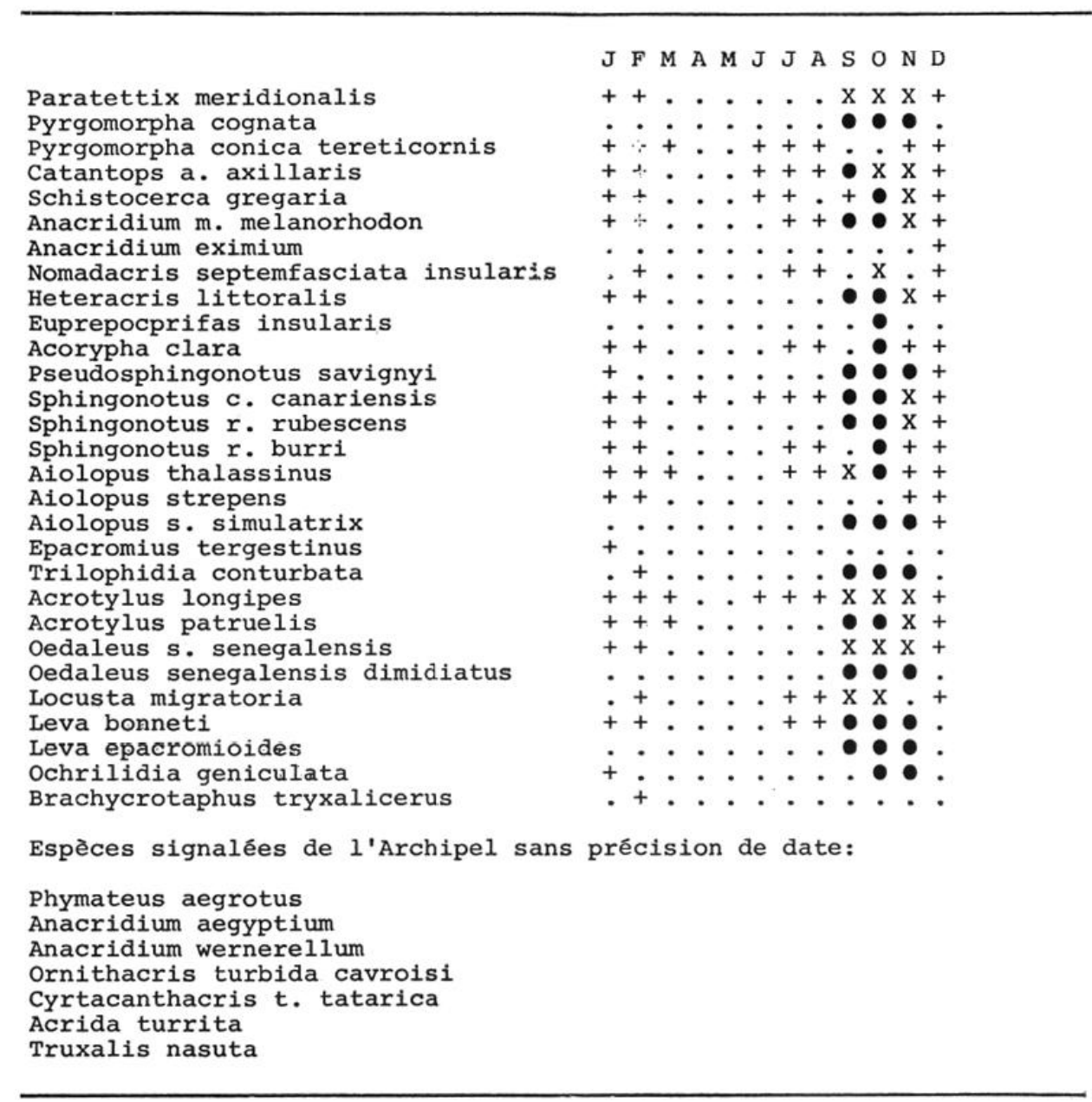

\footnotetext{
Tableau II. Signalisations mensuelles des Acridiens de l'Archipel du Cap-Vert.
}

J à D, mo1s; ., espèce non signalee; + , espèce signalée non retrouvøe? $X$, signalisation confirmee; $\bullet$, signalisation nouvelle.

Parmi ces 23 taxa, trois signalisations seulement sont nouvelles pour l'Archipel. La première est $A$. s. simulator précédemment mentionnée. La seconde constitue de loin la découverte la plus intéressante. Il s'agit, en effet, d'un Eyprepocnemidinae brachyptère, entièrement inconnu, ayant nécessité la création par le déterminateur - M. Donskoff, du Muséum de Paris - d'un genre nouveau : Eyprepocprifas insularis. Enfin, nous avions signalé pour la première fois dans l'Archipel, en 1980, la présence de Pseudosphingonotus savignyi (Duranton et al. 1980). Cette pré- 
sence a été confirmée par Harz (1982) et l'on ne peut qu'être surpris que cette espèce n'ait pas été découverte plus tôt car elle est à la fois fréquente $(28 \%$ des relevés, observée dans 5 îles sur 7), abondante (sa densité moyenne est de 277 imagos par hectare) et trìs caractéristique.

Pour certaines îles, d'assez nombreuses signalisations nouvelles ont été effectuées. C'est le cas pour Schistocerca gregaria, connu de quelques îles et que nous avons trouvé également sur San Antao, San Nicolau et Maio, ainsi que pour Sphingonotus rubescens et Leva bonneti, en particulier.

\section{Caractéristiques principales des peuplements acridiens.}

\subsection{Répartition.}

D'une manière générale, la distribution des espèces sur les différentes îles de l'Archipel semble relever de deux facteurs complémentaires: l'un statique, l'existence de biotopes potentiellement favorables, l'autre dynamique, l'irrégularité fondamentale de la pluviosité d'une année à l'autre.

Ainsi, Schistocerca gregaria se rencontre uniquement dans les biotopes hyperxérotrophes, sableux, souvent dunaires, qui n'existent ni sur Fogo, ni sur Brava, d'où cette espèce est de ce fait exclue. Inversement, la présence de quelques hectares de dunes sur San Antao suffit à permettre l'implantation du Criquet pélerin sur cette île où il n'avait pas été signalé avant 1981.

Ochrilidia geniculata se trouve cantonné dans les touffes des milieux sableux, dunaires, des îles basses, Boa-Vista et Maio.

Paratettix meridionalis est étroitement inféodé aux biotopes hygrotrophes avec eau libre permanente tout au long de l'année et si nous n'avons pu observer cette espèce ni à San Vicente, ni à San Nicolau, ni à Boa-Vista - où elle était pourtant signalée antérieurement - c'est vraisemblablement faute d'avoir pu prospecter sur ces îles les biotopes adéquats qui sont en général fort peu étendus et difficiles à découvrir.

Par contre, dans certaines îles, quelques espèces sont absentes alors même que des biotopes favorables paraissent exister. Ainsi, Pseudosphingonotus savignyi n'a pas été observé sur San Antao et San Vicente bien qu'il soit présent, parfois en très grand nombre, sur toutes les autres îles prospectées dans les milieux pierreux xérotrophes. Aiolopus thalassinus, qui se trouve dans les savanes graminéennes d'altitude sur les îles hautes, n'a pas été capturé sur San Nicolau où certains biotopes leur semblent pourtant propices (et où l'espèce avait d'ailleurs déjà été signalée). Trilophidia conturbata, espèce hygro-géophile, n'a été signalé qu'à Santiago, Maio et Fogo alors que toutes nos observations semblaient indiquer qu'on aurait pu également la trouver sur d'autres îles. L'hypothèse la plus probable est, qu'à ce niveau, le caractère très aléatoire des conditions écologiques (la pluviométrie en particulier) peut provoquer d'une annće à l'autre des modifications impor. tantes de l'abondance relative des espèces sur les différentes îles. A la limite, certains Acridiens peuvent être éliminés purement et simplement de leurs biotopes habituels, voire de l'ensemble d'une île ; la recolonisation paraît ensuite aléatoire et doit s'effectuer par voie aérienne à partir du reste de l'Archipel ou du continent.

Dans cette optique, un cas particulièrement remarquable est celui de Acorypha clara dont la distribution dans l'Archipel semble avoir considérablement évolué depuis les dernières prospections intensives, c'est-à-dire celles menćes par l'expédition Lindberg en 1953-54. Cette espèce était en effet, à l'époque, signalée de toutes 
les îles à l'exclusion de San Antao. Or, malgré l'acuité de notre échantillonnage, elle n'a été retrouvće qu'en cinq localités : 2 sur Santiago et 3 sur Fogo.

L'extrême variabilité saisonnic̀re des contraintes mésologiques paraît donc provoquer des modifications importantes dans la distribution des espèces au sein de l'Archipel, au moins pour celles, plus fragiles, ayant des exigences écologiques strictes; les espèces plus ou moins ubiquistes, présentes sur toutes les îles et sur chacune dans 70 à $80 \%$ des relevés, pouvant trouver plus facilement des zones refuges selon les conditions du moment.

\subsection{Abondance.}

L'abondance, ni même la fréquence, des diverses espèces acridiennes peuplant les îles de l'Archipel, n'ont à ce jour été mentionnées. Il est donc intéressant d'en faire ici état même si les chiffres qui sont avancés ne peuvent l'être qu'à titre indicatif. En effet, ces chiffres ont été obtenus au cours de trois années successives et l'on sait, en particulier au vu des considérations précédentes, que la densité d'une même espèce est éminemment variable d'une année à la suivante. On aura là, néanmoins, une idée moyenne de la plus ou moins grande rareté des Acridiens de l'Archipel à l'époque des prospections (fig. 1 et tabl. III).

Cinq espèces seulement sont à la fois très fréquentes et très abondantes, présentes dans plus de $60 \%$ des relevés et constituant $78 \%$ de l'effectif global des Acridiens. Il s'agit de Oedaleus senegalensis (recensé dans $79 \%$ des relevés et, de loin, l'espèce la plus abondante de l'Archipel dont c'est aussi le principal ravageur), Pyrgomorpha cognata, Sphingonotus rubescens, Acrotylus longipes et Catantops axillaris. On trouve ensuite un ensemble de quatre espèces fréquentes, présentes dans 28 à $36 \%$ des relevés: A. patruelis, A. simulator, $P$. savignyi et $S$. canariensis. Cinq espèces sont présentes dans 11 à $13 \%$ ó des relevés: H. littoralis, L. bonneti, L. epacromioides, $T$. conturbata et $A$. thalassinus. Toutes les autres espèces sont rares, présentes dans moins de $5 \%$ des relevés et pour certaines moins de $1 \%$. Parmi ces dernières, il convient de signaler $P$. meridionalis extrêmement localisé, mais pouvant être très abondant dans ses biotopes avec des densités de plus de 3000 imagos par hectare. Toutes ces dernières espèces, à exigences écologiques plus strictes, sont fort intéressantes dans la mesure où elles constituent des indicateurs de conditions mésologiques bien spécifiques. L'analyse de l'écologie de l'ensemble de ces espèces fera l'objet de publications ultérieures.

\section{Affinités biogéographiques et origine du peuplement.}

Les 621 relevés effectués dans les sept îles principales confirment les conclusions formulées par Chopard quant aux affinités biogéographiques de la faune acridiennes des Iles du Cap-Vert qui est presque entièrement d'origine éthiopienne avec un endémisme très faible (Chopard $19461958 b$ ). Elle se démarque en cela nettement, d'une part, de l'ensemble des autres Iles Macaronćsiennes dont la faune acridienne est essentiellement méditerranéenne et se signale de plus par une forte proportion d'endémiques (16 Acridiens sur 37 aux Canaries) (Chopard 1946), d'autre part, des Iles du Golfe de Guinée où l'endémisme est également très marqué (Chopard $1958 c$ ).

Pour les Iles du Cap-Vert, l'endémisme se limite à un scul genre, Eyprepocprifas (espèce insularis), à deux sous-espèces, Nomadacris septemfasciata insularis et Sphingonotus rubescens burri (dont le statut de sous-espèce mériterait d'ailleurs d'être confirmé) et à une variété insulaire, Oedaleus senegalensis var. dimidiatus. 


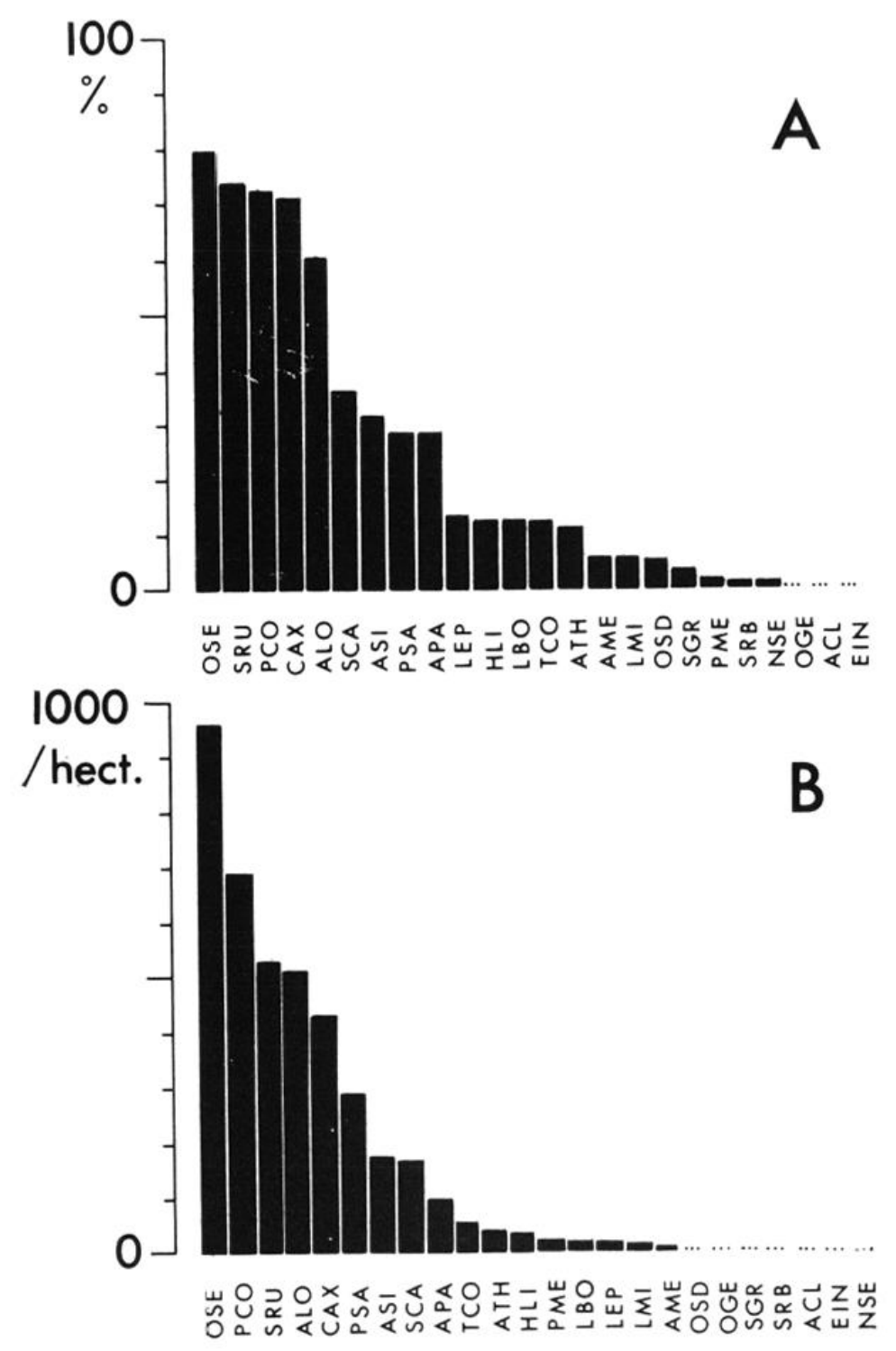

Fig. 1, fréquence (A) et densité à l'hectare moyenne (B) des diverses espèces acridiennes (à l'état imaginal) pour l'ensemble des 621 relevés effectués dans l'Archipel du Cap-Vert.

ACL. Acorypha clara: ALO, Acrotylus longipes; AME, Anacridium $m$. melanorhodon; APA, Acrotylus patruelis; ASI, Aiolopus s. simulator; ATH, Aiolopus thalassinus; CAX. Catantops a. axillaris; EIN, Eyprepocprifas insularis; HLI, Heteracris littoralis; LBO, Leva bonneti: LEP, Leva epacromioides; LMI. Locusta migratoria: NSE, Nomadacris septemfasciata insularis; OGE, Ochrilidia geniculata; OSD, Oedaleus senegalensis var. dimidiatus; OSE, Oedaleus s. senegalensis: PCO, Pyrgomorpha cognata; PME, Paratettix meridionalis; PSA, Pseudosphingonotus savignyi; SCA, Sphingonotus c. canariensis; SGR, Schistocerca gregaria; SRU, Sphingonotus $r$. rubescens; SRB, Sphingonotus rubescens burri; TCO, Trilophidia conturbata.

La plupart des Acridiens de l'Archipel sont des espèces très répandues d'Afrique occidentale à une latitude voisine de celle des Iles, c'est-à-dire au niveau des zones sahćlienne et soudanienne (tabl. IV).

Eyprepocprifas insularis constitue le seul cas d'endémisme absolument certain. Cette espèce, dont l'aspect est celui d'un Acridien de zone forestière au brachyptérisme peut-être encore mal fixé (Donskoff comm. pers.), est incapable d'une introduction active récente et l'on ne peut d'ailleurs la rattacher à aucune espèce 
connue d'Afrique. Une introduction par l'homme à une époque historique paraît exclue pour la même raison. Cette espèce n'a pu vraisemblablement coloniser ces îles qu'à une époque fort reculée, soit par voie terrestre à la faveur d'une liaison ancienne entre l'Archipel et le continent, soit par vol, son brachyptérisme étant alors d'acquisition plus récente. Quoi qu'il en soit, cette espèce paraît constituer une relique d'une faune forestière ayant occupé les îles à une époque reculée, éventuellement lorsque l'Archipel entretenait des relations directes avec le continent africain. La disparition de cette faune primitive aurait été provoquée par le dessè-

Tableau III. Fréquence relative et abondance des diverses espèces acridiennes de l'Archipel du Cap-Vert.

F, fréquence relative; $D$, densité à l'hectare; $M$, densité maximale observée; $A C L$. ALO ... espèces acridiennes (cf. fig. 2) ; $\mathrm{L}$, larves ; I, imagos; $T$, totaux. Nombre de relevés : San Antao, 141; San Vicente, 40; San Nicolau, 100; Boa Vista, 60 ; Maio, 52; Santiago, 165 ; Fogo, 63 .

\begin{tabular}{|c|c|c|c|c|c|c|c|c|c|c|c|c|c|c|c|c|c|c|c|c|c|c|}
\hline & \multirow{2}{*}{\multicolumn{2}{|c|}{$\begin{array}{l}\text { SAN } \\
\mathrm{F}\end{array}$}} & \multicolumn{2}{|c|}{ N ANTAO } & \multirow{2}{*}{$\begin{array}{l}\text { SAN } \\
\text { F }\end{array}$} & \multicolumn{2}{|c|}{ VICENTE } & \multirow{2}{*}{$\begin{array}{l}\text { SAN } \\
F\end{array}$} & \multicolumn{2}{|c|}{ NICOLAU } & \multicolumn{3}{|c|}{ BOA VISTA } & \multicolumn{2}{|c|}{ MAIO } & \multirow[b]{2}{*}{$\mathrm{M}$} & \multicolumn{3}{|c|}{ SANTIAGO } & \multicolumn{2}{|c|}{ FOGO } & \multirow[b]{2}{*}{ M } \\
\hline & & & D & M & & D & M & & D & M & $\mathrm{F}$ & D & M & $\mathrm{F}$ & D & & F & D & M & F & D & \\
\hline ACL & $\begin{array}{l}\text { L } \\
\text { I }\end{array}$ & $\cdot$ & : & • & : & : & . & · & : & : & : & : & : & : & : & : & 1 & 1 & $\begin{array}{r}180 \\
.\end{array}$ & $\begin{array}{l}5 \\
2\end{array}$ & $\begin{array}{r}87 \\
7\end{array}$ & $\begin{array}{r}5310 \\
420\end{array}$ \\
\hline ADOO & $\begin{array}{l}\text { L } \\
\text { I }\end{array}$ & $\begin{array}{l}43 \\
42\end{array}$ & $\begin{array}{l}143 \\
106\end{array}$ & $\begin{array}{l}1410 \\
1290\end{array}$ & $\begin{array}{l}45 \\
75\end{array}$ & $\begin{array}{l}112 \\
272\end{array}$ & $\begin{array}{l}1100 \\
1540\end{array}$ & $\begin{array}{l}79 \\
76\end{array}$ & $\begin{array}{l}522 \\
555\end{array}$ & $\begin{array}{l}6100 \\
7570\end{array}$ & $\begin{array}{l}20 \\
17\end{array}$ & $\begin{array}{l}53 \\
36\end{array}$ & $\begin{array}{r}1320 \\
560\end{array}$ & $\begin{array}{l}31 \\
42\end{array}$ & $\begin{array}{r}62 \\
184\end{array}$ & $\begin{array}{l}1000 \\
1500\end{array}$ & $\begin{array}{l}62 \\
81\end{array}$ & $\begin{array}{r}568 \\
1052\end{array}$ & $\begin{array}{r}6400 \\
13800\end{array}$ & $\begin{array}{l}60 \\
76\end{array}$ & $\begin{array}{l}369 \\
651\end{array}$ & $\begin{array}{l}3460 \\
8790\end{array}$ \\
\hline AME & $\begin{array}{l}\text { L } \\
\text { I }\end{array}$ & $\begin{array}{l}3 \\
4\end{array}$ & $\begin{array}{l}3 \\
1\end{array}$ & $\begin{array}{r}320 \\
60\end{array}$ & $\begin{array}{l}3 \\
5\end{array}$ & $\begin{array}{r}3 \\
11\end{array}$ & $\begin{array}{l}100 \\
390\end{array}$ & $\begin{array}{l}5 \\
2\end{array}$ & $\begin{array}{r}30 \\
1\end{array}$ & $\begin{array}{r}2570 \\
50\end{array}$ & $\stackrel{2}{\cdot}$ & $\begin{array}{l}1 \\
\cdot\end{array}$ & $\begin{array}{r}50 \\
.\end{array}$ & $\begin{array}{r}4 \\
13\end{array}$ & $\begin{array}{l}5 \\
4\end{array}$ & $\begin{array}{r}260 \\
70\end{array}$ & $\begin{array}{l}7 \\
6\end{array}$ & $\begin{array}{r}12 \\
6\end{array}$ & $\begin{array}{l}520 \\
380\end{array}$ & $\begin{array}{l}24 \\
13\end{array}$ & $\begin{array}{r}47 \\
5\end{array}$ & $\begin{array}{r}1130 \\
80\end{array}$ \\
\hline APA & $\begin{array}{l}\text { L } \\
\text { I }\end{array}$ & & $\begin{array}{l}98 \\
49\end{array}$ & $\begin{array}{r}1280 \\
900\end{array}$ & $\begin{array}{l}10 \\
10\end{array}$ & $\begin{array}{l}36 \\
45\end{array}$ & $\begin{array}{l}830 \\
780\end{array}$ & $\begin{array}{r}8 \\
14\end{array}$ & $\begin{array}{r}9 \\
16\end{array}$ & $\begin{array}{l}190 \\
280\end{array}$ & $\begin{array}{l}3 \\
3\end{array}$ & $\begin{array}{r}13 \\
9\end{array}$ & $\begin{array}{l}720 \\
500\end{array}$ & $\begin{array}{l}13 \\
29\end{array}$ & $\begin{array}{l}34 \\
74\end{array}$ & $\begin{array}{l}910 \\
700\end{array}$ & $\begin{array}{l}26 \\
48\end{array}$ & $\begin{array}{l}224 \\
248\end{array}$ & $\begin{array}{l}7780 \\
4610\end{array}$ & $\begin{array}{l}33 \\
38\end{array}$ & $\begin{array}{r}107 \\
68\end{array}$ & $\begin{array}{r}2620 \\
610\end{array}$ \\
\hline ASI & $\begin{array}{l}\text { L } \\
\text { I }\end{array}$ & $\begin{array}{r}11 \\
6\end{array}$ & $\begin{array}{l}13 \\
10\end{array}$ & $\begin{array}{l}540 \\
700\end{array}$ & $\begin{array}{r}3 \\
10\end{array}$ & $\begin{array}{l}5 \\
8\end{array}$ & $\begin{array}{l}180 \\
130\end{array}$ & $\begin{array}{l}35 \\
37\end{array}$ & $\begin{array}{r}72 \\
124\end{array}$ & $\begin{array}{l}1790 \\
5000\end{array}$ & : & : & : & $\begin{array}{l}2 \\
.\end{array}$ & $\begin{array}{l}<1 \\
.\end{array}$ & $\begin{array}{r}10 \\
.\end{array}$ & $\begin{array}{l}32 \\
78\end{array}$ & $\begin{array}{r}98 \\
529\end{array}$ & $\begin{array}{l}1870 \\
6990\end{array}$ & $\begin{array}{r}5 \\
27\end{array}$ & $\begin{array}{r}4 \\
30\end{array}$ & $\begin{array}{l}220 \\
750\end{array}$ \\
\hline ATH & $\begin{array}{ll}\text { L } & 1 \\
\text { I } & 1\end{array}$ & $\begin{array}{l}15 \\
14\end{array}$ & $\begin{array}{l}72 \\
27\end{array}$ & $\begin{array}{r}1830 \\
780\end{array}$ & $\begin{array}{r}8 \\
10\end{array}$ & $\begin{array}{r}207 \\
90\end{array}$ & $\begin{array}{l}7250 \\
3230\end{array}$ & : & . & : & : & : & • & : & : & : & $\begin{array}{l}10 \\
22\end{array}$ & $\begin{array}{l}55 \\
58\end{array}$ & $\begin{array}{l}2210 \\
2250\end{array}$ & $\begin{array}{r}3 \\
10\end{array}$ & $\begin{array}{l}12 \\
44\end{array}$ & $\begin{array}{r}690 \\
2500\end{array}$ \\
\hline CAX & $\begin{array}{l}\text { L } 8 \\
\text { I } 8\end{array}$ & $\begin{array}{l}84 \\
87\end{array}$ & $\begin{array}{l}794 \\
401\end{array}$ & $\begin{array}{r}12370 \\
7180\end{array}$ & $\begin{array}{l}45 \\
85\end{array}$ & $\begin{array}{l}265 \\
734\end{array}$ & $\begin{array}{l}3010 \\
3050\end{array}$ & $\begin{array}{l}90 \\
73\end{array}$ & $\begin{array}{l}818 \\
451\end{array}$ & $\begin{array}{r}10040 \\
4960\end{array}$ & $\begin{array}{r}42 \\
7\end{array}$ & $\begin{array}{r}69 \\
8\end{array}$ & $\begin{array}{r}1500 \\
320\end{array}$ & $\begin{array}{l}21 \\
54\end{array}$ & $\begin{array}{r}32 \\
155\end{array}$ & $\begin{array}{r}440 \\
1420\end{array}$ & $\begin{array}{l}80 \\
76\end{array}$ & $\begin{array}{l}585 \\
380\end{array}$ & $\begin{array}{r}11470 \\
7550\end{array}$ & $\begin{array}{l}81 \\
84\end{array}$ & $\begin{array}{r}1129 \\
922\end{array}$ & $\begin{array}{l}11840 \\
12680\end{array}$ \\
\hline EIN & $\begin{array}{l}\text { L } \\
\text { I }\end{array}$ & : & : & : & : & : & : & i & $<i$ & 20 & : & $\dot{.}$ & : & . & : & : & $\dot{5}$ & : & : & : & : & $\cdot$ \\
\hline HLI & $\begin{array}{l}\text { L } \\
\text { I } 1\end{array}$ & $\begin{array}{r}8 \\
16\end{array}$ & $\begin{array}{l}18 \\
33\end{array}$ & $\begin{array}{r}680 \\
1290\end{array}$ & $\begin{array}{l}53 \\
68\end{array}$ & $\begin{array}{l}152 \\
288\end{array}$ & $\begin{array}{l}1700 \\
1540\end{array}$ & $\begin{array}{l}2 \\
5\end{array}$ & $\begin{array}{l}5 \\
6\end{array}$ & $\begin{array}{l}370 \\
240\end{array}$ & $\begin{array}{l}15 \\
20\end{array}$ & $\begin{array}{l}18 \\
18\end{array}$ & $\begin{array}{l}320 \\
330\end{array}$ & 10 & 7 & $150^{\circ}$ & $\begin{array}{l}2 \\
2\end{array}$ & $\begin{array}{l}8 \\
2\end{array}$ & $\begin{array}{l}700 \\
210\end{array}$ & : & : & : \\
\hline LBO & $\begin{array}{l}\text { L } 2 \\
\text { I }\end{array}$ & $\begin{array}{r}27 \\
9\end{array}$ & $\begin{array}{r}119 \\
15\end{array}$ & $\begin{array}{l}5280 \\
1070\end{array}$ & $\dot{ }$ & $\dot{.}$ & $\dot{.}$ & $\begin{array}{l}14 \\
11\end{array}$ & $\begin{array}{l}25 \\
10\end{array}$ & $\begin{array}{l}500 \\
270\end{array}$ & : & $\dot{.}$ & . & $\begin{array}{l}2 \\
2\end{array}$ & $\begin{array}{l}2 \\
6\end{array}$ & $\begin{array}{r}90 \\
300\end{array}$ & $\begin{array}{l}19 \\
22\end{array}$ & $\begin{array}{l}36 \\
26\end{array}$ & $\begin{array}{r}1870 \\
930\end{array}$ & $\begin{array}{l}38 \\
17\end{array}$ & $\begin{array}{r}216 \\
14\end{array}$ & $\begin{array}{r}3270 \\
300\end{array}$ \\
\hline LEP & $\begin{array}{ll}\text { L } & 1 \\
\text { I } & 1\end{array}$ & $\begin{array}{l}17 \\
10\end{array}$ & $\begin{array}{l}84 \\
12\end{array}$ & $\begin{array}{r}7440 \\
610\end{array}$ & . & : & : & $\begin{array}{l}9 \\
8\end{array}$ & $\begin{array}{l}9 \\
9\end{array}$ & $\begin{array}{l}190 \\
300\end{array}$ & : & : & : & $\begin{array}{l}4 \\
2\end{array}$ & $\begin{array}{l}7 \\
1\end{array}$ & $\begin{array}{r}270 \\
30\end{array}$ & $\begin{array}{l}13 \\
25\end{array}$ & $\begin{array}{l}52 \\
31\end{array}$ & $\begin{array}{l}4810 \\
1210\end{array}$ & $\begin{array}{l}38 \\
21\end{array}$ & $\begin{array}{r}131 \\
16\end{array}$ & $\begin{array}{r}2700 \\
220\end{array}$ \\
\hline LMI & $\begin{array}{ll}\text { L } & 1 \\
\text { I } & 1\end{array}$ & $\begin{array}{l}13 \\
11\end{array}$ & $\begin{array}{l}17 \\
18\end{array}$ & $\begin{array}{l}500 \\
490\end{array}$ & : & : & : & : & : & $\dot{.}$ & : & : & : & : & : & : & : & : & : & $\begin{array}{l}10 \\
27\end{array}$ & $\begin{array}{l}11 \\
54\end{array}$ & $\begin{array}{l}340 \\
750\end{array}$ \\
\hline NSE & $\begin{array}{l}\text { L } \\
\text { I }\end{array}$ & $\dot{.}$ & $\dot{.}$ & : & : & : & $\dot{.}$ & $\dot{.}$ & : & : & : & : & : & : & . & : & : & : & $\dot{.}$ & $\begin{array}{l}5 \\
6\end{array}$ & $\begin{array}{r}14 \\
4\end{array}$ & $\begin{array}{l}680 \\
150\end{array}$ \\
\hline OGE & $\begin{array}{l}\text { L } \\
\text { I }\end{array}$ & $\dot{.}$ & : & : & : & : & : & : & : & : & 2 & $\begin{array}{l}5 \\
.\end{array}$ & $\begin{array}{r}270 \\
.\end{array}$ & $\begin{array}{l}6 \\
4\end{array}$ & $\begin{array}{l}51 \\
11\end{array}$ & $\begin{array}{r}1360 \\
450\end{array}$ & : & : & : & : & : & : \\
\hline OSD & $\begin{array}{l}\mathrm{L} \\
\mathrm{I}\end{array}$ & $\dot{5}$ & i & $4 \dot{0}$ & : & : & $\therefore$ & $\dot{6}$ & $\dot{4}$ & $14 \dot{0}$ & 10 & $\dot{4}$ & 90 & $\dot{2}$ & $<\mathrm{i}$ & $2 \dot{0}$ & : & : & : & 10 & $\dot{9}$ & $300^{\circ}$ \\
\hline OSE & $\begin{array}{l}\text { L } \\
\text { I }\end{array}$ & $\begin{array}{l}28 \\
83\end{array}$ & $\begin{array}{r}175 \\
1031\end{array}$ & $\begin{array}{r}7810 \\
14840\end{array}$ & $\begin{array}{l}23 \\
55\end{array}$ & $\begin{array}{r}32 \\
310\end{array}$ & $\begin{array}{r}240 \\
2410\end{array}$ & $\begin{array}{l}15 \\
76\end{array}$ & $\begin{array}{r}22 \\
583\end{array}$ & $\begin{array}{r}480 \\
7580\end{array}$ & $\begin{array}{l}10 \\
97\end{array}$ & $\begin{array}{r}5 \\
3062\end{array}$ & $\begin{array}{r}140 \\
22070\end{array}$ & $\begin{array}{r}4 \\
50\end{array}$ & $\begin{array}{r}29 \\
247\end{array}$ & $\begin{array}{l}1350 \\
2160\end{array}$ & $\begin{array}{l}56 \\
87\end{array}$ & $\begin{array}{l}179 \\
781\end{array}$ & $\begin{array}{l}2570 \\
9180\end{array}$ & $\begin{array}{l}19 \\
78\end{array}$ & $\begin{array}{r}29 \\
707\end{array}$ & $\begin{array}{r}370 \\
11980\end{array}$ \\
\hline $\mathrm{PCO}$ & $\begin{array}{l}\text { L } \\
\text { I }\end{array}$ & $\begin{array}{l}70 \\
70\end{array}$ & $\begin{array}{l}507 \\
399\end{array}$ & $\begin{array}{l}4650 \\
3090\end{array}$ & $\begin{array}{l}68 \\
83\end{array}$ & $\begin{array}{l}169 \\
444\end{array}$ & $\begin{array}{l}1150 \\
2790\end{array}$ & $\begin{array}{l}69 \\
73\end{array}$ & $\begin{array}{l}317 \\
232\end{array}$ & $\begin{array}{l}6450 \\
6070\end{array}$ & $\begin{array}{l}28 \\
23\end{array}$ & $\begin{array}{l}43 \\
23\end{array}$ & $\begin{array}{l}800 \\
270\end{array}$ & $\begin{array}{l}54 \\
63\end{array}$ & $\begin{array}{r}163 \\
90\end{array}$ & $\begin{array}{r}1440 \\
860\end{array}$ & $\begin{array}{l}88 \\
96\end{array}$ & $\begin{array}{l}1486 \\
1851\end{array}$ & $\begin{array}{l}14890 \\
12900\end{array}$ & $\begin{array}{l}60 \\
60\end{array}$ & $\begin{array}{l}189 \\
209\end{array}$ & $\begin{array}{l}2210 \\
1330\end{array}$ \\
\hline PME & $\begin{array}{l}\text { L } \\
\text { I }\end{array}$ & $\begin{array}{l}1 \\
5\end{array}$ & $\begin{array}{r}2 \\
15\end{array}$ & $\begin{array}{l}120 \\
760\end{array}$ & : & : & : & : & : & : & : & : & $\dot{ }$ & $\begin{array}{l}2 \\
2\end{array}$ & $\begin{array}{l}14 \\
48\end{array}$ & $\begin{array}{r}710 \\
2500\end{array}$ & $\begin{array}{l}1 \\
1\end{array}$ & $\begin{array}{r}9 \\
27\end{array}$ & $\begin{array}{l}1300 \\
2770\end{array}$ & $\dot{.}$ & : & : \\
\hline PSA & I & : & : & : & : & : & • & $\begin{array}{r}6 \\
14 \\
\end{array}$ & $\begin{array}{r}9 \\
25\end{array}$ & $\begin{array}{l}340 \\
590\end{array}$ & $\begin{array}{l}42 \\
92\end{array}$ & $\begin{array}{r}201 \\
1894\end{array}$ & $\begin{array}{l}10500 \\
36930\end{array}$ & $\begin{array}{l}17 \\
73\end{array}$ & $\begin{array}{r}37 \\
555\end{array}$ & $\begin{array}{l}1120 \\
3290\end{array}$ & $\begin{array}{l}16 \\
38\end{array}$ & $\begin{array}{r}21 \\
143\end{array}$ & $\begin{array}{r}460 \\
4530\end{array}$ & $\begin{array}{l}5 \\
8\end{array}$ & $\begin{array}{l}18 \\
53\end{array}$ & $\begin{array}{r}850 \\
2790\end{array}$ \\
\hline SCA & $\begin{array}{ll}\text { L } & 1 \\
\text { I } & 3\end{array}$ & $\begin{array}{l}13 \\
31\end{array}$ & $\begin{array}{l}42 \\
81\end{array}$ & $\begin{array}{l}1440 \\
1730\end{array}$ & $\begin{array}{r}5 \\
25\end{array}$ & $\begin{array}{r}3 \\
98\end{array}$ & $\begin{array}{r}100 \\
1700\end{array}$ & $\begin{array}{r}7 \\
24\end{array}$ & $\begin{array}{l}10 \\
62\end{array}$ & $\begin{array}{r}420 \\
1150\end{array}$ & $\begin{array}{r}7 \\
13\end{array}$ & $\begin{array}{r}2 \\
22\end{array}$ & $\begin{array}{r}80 \\
770\end{array}$ & $1 \dot{9}$ & 18 & $350^{\circ}$ & $\begin{array}{l}25 \\
62\end{array}$ & $\begin{array}{r}79 \\
410\end{array}$ & $\begin{array}{l}5370 \\
5790\end{array}$ & $\begin{array}{l}24 \\
38\end{array}$ & $\begin{array}{r}51 \\
142\end{array}$ & $\begin{array}{r}870 \\
2730\end{array}$ \\
\hline SGR & $\begin{array}{l}\mathrm{L} \\
\mathrm{I}\end{array}$ & i & $<\dot{i}$ & 30 & $\dot{8}$ & 3 & $80^{\circ}$ & $\dot{6}$ & $\dot{2}$ & 70 & 8 & $\dot{4}$ & 110 & 12 & $\dot{3}$ & 30 & $\dot{0}$ & $\dot{ }$ & : & $\dot{.}$ & : & : \\
\hline SRB & $\begin{array}{l}\text { L } \\
\text { I }\end{array}$ & i & $\dot{2}$ & $170^{\circ}$ & : & $\dot{ }$ & : & $\dot{5}$ & $\dot{3}$ & $150^{\circ}$ & i & $\dot{3}$ & $200^{\circ}$ & $\therefore$ & : & i & : & . & : & : & : & : \\
\hline SRU & $\begin{array}{l}\text { L } \\
\text { I }\end{array}$ & $\begin{array}{l}56 \\
64\end{array}$ & $\begin{array}{l}206 \\
650\end{array}$ & $\begin{array}{l}2920 \\
5200\end{array}$ & $\begin{array}{l}48 \\
85\end{array}$ & $\begin{array}{r}69 \\
475\end{array}$ & $\begin{array}{r}610 \\
2360\end{array}$ & $\begin{array}{l}61 \\
84\end{array}$ & $\begin{array}{l}182 \\
955\end{array}$ & $\begin{array}{l}1950 \\
3600\end{array}$ & $\begin{array}{l}33 \\
78\end{array}$ & $\begin{array}{r}30 \\
262\end{array}$ & $\begin{array}{r}320 \\
1550\end{array}$ & $\begin{array}{l}33 \\
71\end{array}$ & $\begin{array}{r}46 \\
328\end{array}$ & $\begin{array}{r}370 \\
2370\end{array}$ & $\begin{array}{l}27 \\
76\end{array}$ & $\begin{array}{r}43 \\
441\end{array}$ & $\begin{array}{r}940 \\
2960\end{array}$ & $\begin{array}{l}37 \\
57\end{array}$ & $\begin{array}{r}89 \\
198\end{array}$ & $\begin{array}{l}1940 \\
1150\end{array}$ \\
\hline TCO & $\begin{array}{l}\text { L } \\
\text { I }\end{array}$ & $\dot{.}$ & $\dot{.}$ & : & $\dot{.}$ & : & : & : & $\dot{.}$ & : & $\dot{ }$ & $\dot{.}$ & $\dot{.}$ & $\begin{array}{l}10 \\
13\end{array}$ & $\begin{array}{r}8 \\
51\end{array}$ & $\begin{array}{r}200 \\
1180\end{array}$ & $\begin{array}{l}15 \\
32\end{array}$ & $\begin{array}{r}51 \\
139\end{array}$ & $\begin{array}{l}1500 \\
2960\end{array}$ & $\begin{array}{r}8 \\
22\end{array}$ & $\begin{array}{l}10 \\
50\end{array}$ & $\begin{array}{r}350 \\
1190\end{array}$ \\
\hline $\mathrm{T}$ & $\begin{array}{l}\text { L } \\
\text { I }\end{array}$ & & $\begin{array}{l}2296 \\
2854\end{array}$ & & & $\begin{array}{l}1053 \\
2778\end{array}$ & & & $\begin{array}{l}2030 \\
3039\end{array}$ & & & $\begin{array}{r}440 \\
5345\end{array}$ & & & $\begin{array}{r}491 \\
1783\end{array}$ & & & $\begin{array}{l}3507 \\
6124\end{array}$ & & & $\begin{array}{l}2513 \\
3183\end{array}$ & \\
\hline
\end{tabular}


chement du climat et l'isolement de l'Archipel. Celui-ci n'aurait pu ensuite être recolonisé que par des apports en provenance du continent à la faveur de vents favorables de secteur Est à Nord-Est. C'est ainsi que peut s'expliquer la présence sur l'Archipel de la quasi totalité des autres espèces que nous avons pu y observer.

De nombreux Acridiens doivent d'ailleurs, encore actuellement, atteindre plus ou moins régulièrement les Iles en provenance du continent voisin. Le phénomène est spectaculaire pour des espèces abondantes, bons voiliers, comme le Criquet pélerin, Schistocerca gregaria, dont les vols d'individus grégaires venant d'Afrique ont été signalés s'abattant sur les Iles à plusicurs reprises (Chevalier 1935, Saraiva 1962). Chez Oedaleus senegalensis, lorsque les effectifs sont nombreux en Afrique de l'Ouest, des individus emportés en mer par les vents de Nord-Est en septembre et octobre ont été repêchés par des navires, parfois assez loin des còtes africaines (plus de $200 \mathrm{~km}$ ) (Ritchic 1978). Il ne fait guère de doute, dans ces conditions, que des individus de cette espèce doivent pouvoir atteindre occasionnellement les îles de l'Archipel du Cap-Vert et que la possibilité de spéciation apparaît de ce fait fort limitée (Ritchie l.c.). Ces phénomènes de recolonisation plus ou moins régulière à partir du continent doivent exister pour toutes les autres espèces macroptères, même si la probabilité en est plus faible pour certaincs.

Tableau IV. Affinités biogéographiques de la faune acridienne de l'Archipel du Cap-Vert.

$\mathrm{CV}$ : Espèces endémiques des îles du Cap-Vert. — AS: Espèces présentes dans les zones sahélienne et soudanienne d'Afrique de l'Ouest. - AG : Espèce signalée de la zone guinéenne d'Afrique de l'Ouest. - AE : Espèce éthiopienne non présente en Afrique de l'Ouest. MM : Espèces présentes dans la région méditerranéenne. - PP : Espèce paléarctique signalée d'Europe centrale et d'Asie.

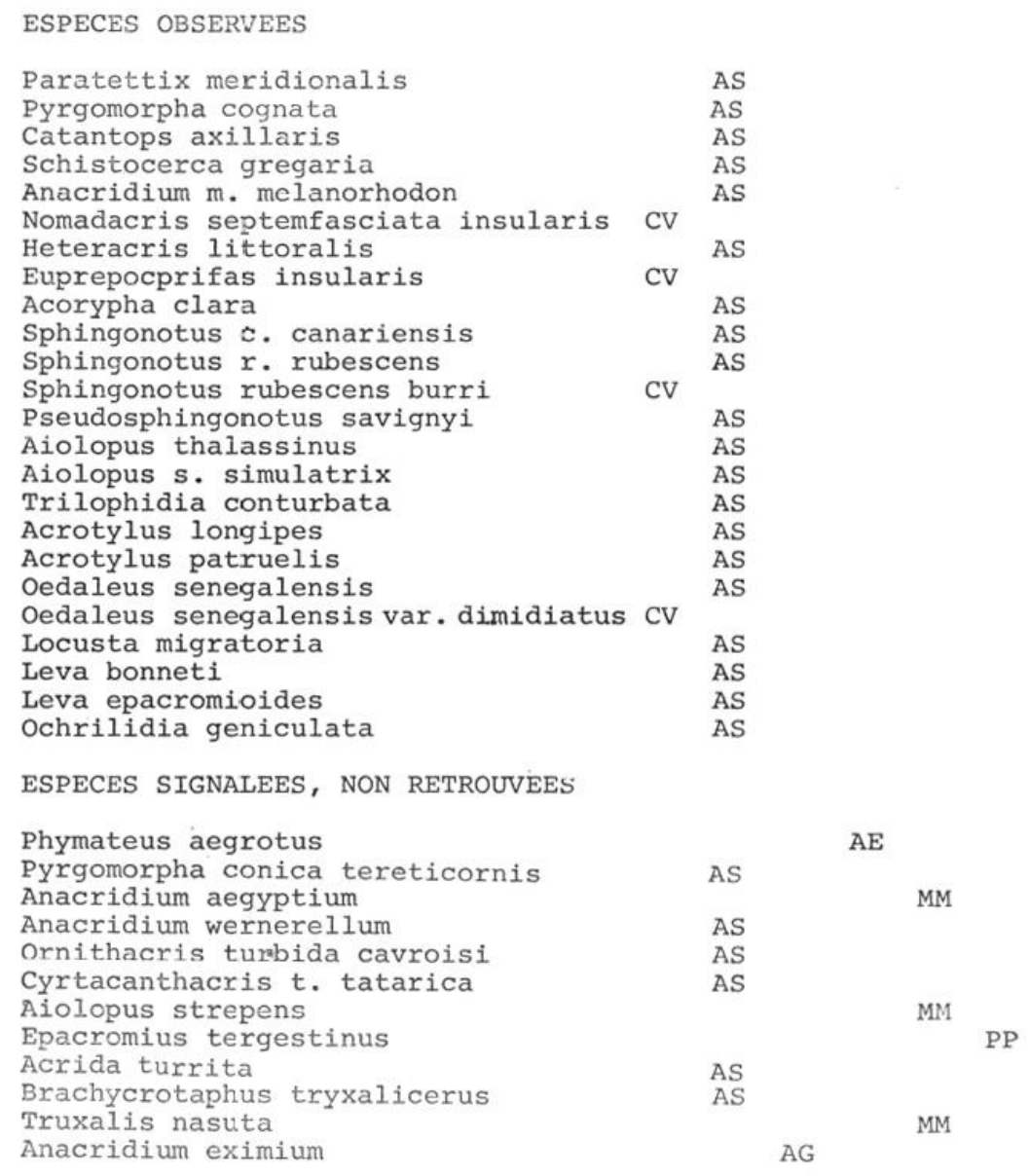




\section{Conclusions.}

Le très abondant matéricl que nous avons pu étudier dans l'Archipel du CapVert confirme pour l'essentiel les conclusions formulées antérieurement par Chopard $(19461958$ b) : la faune acridienne de ces îles est pauvre en espèces, presque entièrement d'origine éthiopienne et présente un endémisme très faiblc.

$\mathrm{Si}$, au niveau de chaque île, de nombreuses signalisations nouvelles ont été effectuées, globalement, pour l'ensemble de l'Archipel, le nombre d'espèces nouvelles est peu élevé, montrant ainsi que cette faune commence a être bien connue en dépit de quelques problèmes de nomenclature et du statut incertain de quelques sous-espèces.

Cependant, la découverte d'un genre nouveau, endémique des Iles du Cap-Vert, prouve que quelques espèces rares et de grand intérêt biogéographique peuvent encore avoir échappé aux investigations, principalement dans les biotopes spécialisés.

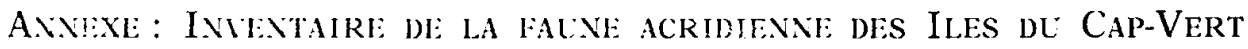

L'inventaire suivant tient compte de nos travaux personnels et de l'ensemble de ceux de nos prédécesseurs sur le sujet: publications ayant trait spécifiquement aux Iles du Cap-Vert complétécs par les signalisations publićes dans les deux catalogues de Johnston $(1956,1968)$ et par l'addenda de Johnsen (1974) à la liste des Acridiens des Iles Macaronésiennes publiće par Chopard en 1954.

Il convient de signaler que la liste récente de Harz (1982) des Orthoptères du Cap-Vert est, au moins en ce qui concerne les Acridiens, très incomplète et nc tient compte ni des travaux de Johnsen $(1974,1977)$ ni de l'important travail de Chopard de $1958 b$.

Concernant nos récoltes, les spécimens de référence se trouvent au Laboratoire d'Entomologie du Muséum national d'Histoire naturclle, à Paris. Des doubles existent dans les collections du Laboratoire de Faunistique du Centre de Recherches GERDAT (2) de Montpellier.

\section{ACRIDOIDEA}

\section{TI:TRIGIDAE}

\section{Paratettix meridionalis (Rambur 1838).}

1833. Tetrix meridionalis Rambur, Faune Andal., vol. II, p. 65.

Cette espèce hyper-hygrophile est largement distribuée, du Sud de l'Europe à Madagascar, à travers toute l'Afrique. Elle est strictement localisée aux abords des points d'eau permanents qui, sauf sur San Antao, sont très rares dans l'Archipel. Nous l'avons trouvée dans $2 \%$ de nos relevés. Elle est signalée de toutes les îles principales à l'exclusion de Sal et de Fogo, ce qui semble correspondre (sous réserve d'investigations plus poussćes) à l'absence sur ces îles de points où l'eau affleure en permanence. Cette espèce peut être localement très abondante et, par 
trois fois, des densités supérieures à 1500 imagos par hectare ont été observées, le maximum étant de 2770 .

\section{PYRGOMORPIIIDAE}

Phymateus aegrotus (Gerstaecker 1869).

1869. Poecilocera aegrota Gerstaecker, Arch. Naturgesch., vol. XXXV, fasc. 1, p. 216.

Cette espèce est seulement mentionnée par Bolivar (1889) et n'a pas été retrouvée depuis. Il s'agit d'un Acridien présent essentiellement en Afrique de l'Est, Centrale et du Sud. La signalisation la plus prochc des Iles du Cap-Vert a été effectuée par Karsch (1888) au Ghana. Il s'agit vraisemblablement d'une introduction accidentelle.

Pyrgomorpha Serville 1838.

Ainsi que le signale Johnsen (1981), les espèces de ce genre sont fort difficiles à déterminer. Le travail de Hsiung \& Kevan (1975) n'est que préliminaire et une détermination exacte des espèces du genre Pyrgomorpha devra nécessiter une révision définitive.

En l'attente, et bien que jusqu'à présent seul $P$. conica tereticornis Brullé (anciennement $P$. tereticornis) ait été signalé de l'Archipel (3) (à l'exception d'une signalisation de $P$. cognata de Bolivar, en 1884, rapportée par Saraiva en 1961), nous adopterons les conclusions de Johnsen (1974) sur les Pyrgomorpha des îles Canaries et nommerons notre matériel $P$. cognata. Nous envisageons ultérieurement d'entreprendre des études de génétique des populations pour préciser le statut de diverses souches africaines.

Pyrgomorpha cognata Krauss 1877.

1877. Pyrgomorpha cognata Krauss, S.B. Akad. Wiss. Wien, vol. LXXVI, fasc. 1, p. 58.

Cette espèce est l'une des plus abondantes et des plus fréquentes de l'Archipel. Présente à l'état imaginal dans $72 \%$ des relevés, sa densité moyenne est de 679 imagos par hectare. Nous l'avons observée dans toutes les îles prospectées. Sur Santiago, sa fréquence atteint $96 \%$ des relevés avec une densité moyenne et une densité maximale, respectivement, de 1850 et de 12900 imagos par hectare. Les larves sont nombreuses et observées à des densités comparables à celles des adultes.

Pyrgomorpha conica tereticornis (Brullé 1840).

syn. P. tereticornis.

1840. Tryxalis tereticornis Brullé, Hist. nat. Iles Can., "Orth.", p. 76.

A ce jour, tous les individus appartenant au genre Pyrgomorpha capturés dans les Iles du Cap-Vert avaient été rattachés à cette sous-espècc (Chopard 19361958 b, Harz 1982). Pour des raisons exposées précćdemment nous rattacherons notre matériel à $P$. cognata.

(3) D'après D.K. McE. Kevan 1981 comm. pers. 


\section{Catantopinae}

Catantops axillaris axillaris (Thunberg 1815).

1815. Gryllus axillaris Thunberg, Mém. Acad. Imp. Sci. St. Pétersb., vol. V, p. 250.

Cette sous-espèce est distribuće dans toute l'Afrique Tropicale et l'Asie du SudOuest. Nous l'avons rencontrée dans toutes les îles visitées. Elle est également signalće de Sal, Brava et Santa Luzia. A la fois fréquente et abondante, elle est rencontrée dans plus de $80 \%$ des relevés sur San Antao, San Vicente et Fogo; dans plus de $50 \%$ de ceux de San Nicolau, Maio et Santiago. C. axillaris est par contre assez rare sur Boa Vista où il n'a été rencontré que dans $7 \%$ des cas. La densité maximale a été notée sur Fogo avec 12680 imagos par hectare.

Cette sous-espèce n'a qu'une seule génération annuelle et une diapause imaginale de saison sèche (Lecoq 1978 1980). A l'époque des prospections, les larves sont encore nombreuses; la plupart des adultes capturés sont de jeunes individus de la nouvelle génération annuelle qui passeront vraisemblablement la saison sèche en diapause imaginale.

\section{Cyrtacanthacridinae}

\section{Schistocerca gregaria (Forskal 1775).}

1775. Gryllus gregarius Forskal, Descr. Anim., p. 81.

On a longtemps pensé que le Criquet pélerin - largement distribué dans toute l'Afrique au Nord de l'équateur, le Sud de l'Europe, le Proche et le Moyen-Orient jusqu'à l'Inde - ne se reproduisait pas aux Iles du Cap-Vert et que seuls des individus grégaires en provenance d'Afrique colonisaient de temps à autres les îles en période d'invasion (Chevalier 1935, Chopard 1936). Selon Chevalier (l.c.), les vols d'individus grégaires sont réputés atteindre l'Archipel essentiellement en septembre et octobre. Entre 1940 et 1975, un essaim a été signalé une fois dans les îles (Pedgley, 1981).

En fait, dès 1958, Chopard notait que, de tous les individus capturés en 1953-54 par l'expédition Lindberg, un seul mâle avait la coloration jaune des imagos sexuellement mûrs et pouvait aroir été importé par vol du continent. Les autres étaient sexuellement immatures et il s'agissait donc d'une présomption quant à une possibilité de reproduction de cette espèce dans les Iles du Cap-Vert.

Pour notre part, nous avons identifié 24 individus de $S$. gregaria. Tous les individus observés étaient de phase solitaire et la densité maximale notée de 110 imagos par hectare. Trois individus seulement ont été attrapés à cause des difficultés de capture de cet Acridien au vol puissant. Ces trois individus se répartissent de la manière suivante: un mâle d'aspect jeune à téguments durs, une femelle jeune en pré-vitellogenèse à téguments durcis également et une femelle jeune à téguments encore mous née très vraisemblablement sur place. Aucune larve n'a été capturée, mais il est propable que lc dévcloppcment larvaire de cette génération - qui doit correspondre à la génération d'été sur le continent africain - s'est déroulé antérieurement à l'époque de nos prospections, c'est-à-dire en juillet, août et septembre. Des larves devraient pouvoir être découvertes à cette époque. D'après G. Popov (1982 comm. pers.), Mle McLeer aurait rapporté en 1975 une larve de Criquet pélerin des Iles du Cap-Vert. 
Jusqu'à ces dernières années, cette espèce n'était signalćc que de San Vicente, Santa Luzia, Sal et Boa Vista. Elle vient d'être découverte sur Santiago (Harz 1982) et pour notre part, en plus de San Vicente et Boa Vista, nous l'avons également observéc sur San Antao, San Nicolau et Maio, soit dans 21 relevés au total. Il s'agit donc d'une espèce peu fréquente mais beaucoup plus largement distribuée au scin de l'Archipel qu'on ne le pensait. En particulier, elle n'est nullement limitée aux seules îles nord-orientales comme le signalait Saraiva (1961), mais se trouve sur toutes les îles susceptibles de lui offrir des milieux sableux, hyper-xérotrophes. Ces conditions sont plus fréquentes sur des îles comme San Vicente, Boa Vista et Maio. Cependant, ces conditions réalisées même sur des superficies restreintes suffisent à permettre l'implantation du Criquet pélerin. Ainsi, sur San Antao, nous avons pu repérer, à l'Ouest de Porto Novo, une zone sableuse de quelques hectares avec 2 ou 3 dunes vives importantes où $S$. gregaria était effectivement présent et où nous avons pu l'observer à deux reprises à quelques jours d'intervalle.

\section{Anacridium aegyptium (Linnaeus 1764).}

17ô4. Gryllus (Locusta) aegyptius Linneəus, Mus. Lud. Ulr. Reg., p. 138.

Ce Criquet arboricole a été signalé par Bolivar en 1889 mais n'a pas été retrouvé depuis. Il s'agit, en fait, d'une espèce constituant un des plus beaux exemples de distribution méditerranécnne (Dirsh \& Uvarov 1953) et cette signalisation peut être considéréc comme douteuse ou tout à fait accidentelle.

\section{Anacridium wernerellum (Karny 1907).}

1907. Locusia (Orthacanthacris) wernerella IKarny, S.B. Akad. Wiss. Wien, vol. CVI, fasc. 1. p. 305.

A. Wernerellum a été signalé des Iles du Cap-Vert par Dirsh \& Uvarov (1953) d'après des exemplaires capturés par Delaunay en 1882, sur San Vicente, exemplaires qui auraient étć déposés au Muséum de Paris mais que nous n’avons pu retrouver.

L'aire de distribution de cette espèce couvre, en Afrique de l'Ouest, la zone soudanienne, c'est-à-dire les savanes les moins arides, et s'incline à l'Est vers la région des grands lacs. Il n'est donc pas illogique de la retrouver dans l'Archipel puisqu'on l'observe, à des latitudes comparables, sur le continent africain (Mauritanie par exemple). Sa présence dans les Iles, si elle s'avère exacte, paraît tout à fait exceptionnelle.

\section{Anacridium melanorhodon melanorhodon (Walker 1870).}

1870. Acridium melanorhodon Walker, Cat. Derm. Salt. Brit. Mus., 3" partie, p. 585.

Cet autre Criquet arboricole, capable de former des essaims, a été décrit des Iles du Cap-Vert par Walker en 1870. Son aire de répartition sur le continent correspond à une étroite bande traversant l'Afrique entre les parallèles 12 et $18^{\circ} \mathrm{N}$, du Cap Vert au Soudar. Celte sous-espèce est donc plus septentrionale que l'espèce précédentc. Toutes deux présentent une seule génération annuelle; les adultes passent la saison sèche en diapause imaginale (Dirsh \& Uvarov 1953, Lecoq 1980).

A. melanorhodon a été obscrvé dans toutes les îles prospectées. Les imagos 
ont été trouvés dans 34 relevés et les larves dans 40 d'entre eux. Cette sous-espèce est assez étroitement inféodéc aux peuplements arbustifs et arborés à base d'épineux comme Acacia farnesiana Wild., Parkinsonia aculeata L. ou Dichrostachys cinerea (L.) Wight et Arn. Elle peut être localement abondante et une densité de 2570 larves par hectare a été observée sur San Nicolau.

En fin de saison des pluies, les larves sont encore nombreuses, relativement âgées. On observe d'assez nombreux imagos à téguments mous qui devraient passer la saison sèche en diapause imaginale et quelques vieux individus de la génération précédente.

\section{Anacridium eximium (Sjöstedt 1918).}

1918. Ortacanthacris eximia Sjöstedt, Ark. Zool., vol. XII, fasc. 1, p. 2, fig. 2.

Cette espèce n'était connue auparavant que par une seule femelle capturée dans la région d'Accra, au Ghana. Considérée pendant un temps comme un synonyme de A. moestum (Rehn 1944), Dirsh \& Uvarov (1953) lui accordent le statut d'une espèce indépendante.

Johnsen (1977) considère qu'un mâle capturé à San Vicente en 1945 par une expédition danoise, correspondrait au mâle de $A$. eximium jusqu'alors inconnu. Le Ghana et les Iles du Cap-Vert sont évidemment fort éloignés, mais les Anacridium sont de bons voiliers, effectuant de nombreux déplacements saisonniers et, par ailleurs, des exemplaires d'Anacridium ont été parfois capturés en mer (Johnsen 1970). L'exemplaire recueilli dans les Iles paraît âgé, avec des ailes postérieures très abimées. Il s'agirait là d'une espèce d'Anacridium uniquement accidentelle dans l'Archipel (Johnsen 1977).

\section{Nomadacris septemfasciata insularis Chopard 1936.} p. 95.

1936. Nomadacris septemfasciata Walk. ssp. insularis Chopard, Rev. franç. Ent., vol. III,

Cette sous-espèce du Criquet nomade africain a été décrite par Chopard, en 1936, sur les exemplaires capturés par Chevalier à Fogo. A l'époque, N. septemfasciata n'était pas connu d'Afrique de l'Ouest où il a depuis été signalé à de nombreuses reprises (Golding 1948, Roblot 1950, Wintrebert 1955, Chopard $1958 a$, Davey et al. 1959, Descamps 1965 1968). Son aire couvre actuellement toute l'Afrique au Sud du Sahara.

Tous les individus que nous avons pu capturer correspondent bien à la sousespèce insularis décrite par Chopard avec, en particulier, une taille réduite et des taches sur les élytres presque complètement effacces. A titre indicatif, des mensurations effectuées sur seulement trois individus ont donné les résultats suivants: $1 q: \mathrm{E}=5.20 \mathrm{~mm}, \mathrm{~F}=3.00 ; 1$ q $\mathrm{E}=5.20, \mathrm{~F}=2.99 ; 1$ o $: \mathrm{E}=3.90, \mathrm{~F}=$ 2.14. Elles correspondent sensiblement aux dimensions fournies par Chopard (1936).

$N$. septemfasciata insularis était signalé de San Antao, Santiago, Fogo et Brava. Nous l'avons retrouvé uniquement sur Fogo, dans 4 relevés et en faibles densités. Tous les individus observés étaient de type solitaire. Les quelques larves capturées correspondent à des individus de la seule et unique génération annuelle. Le cycle est vraisemblablement le même que sur le continent : univoltin avec diapause imaginale de saison sìche. 
Ornithacris turbida cavroisi (Finot 1907).

1907. Acridium cavroisi Finot, Ann. Soc. ent. France, vol. LXXVI, p. 272 et 346.

Cette espèce, commune de l'Afrique occidentale à l'Afrique centrale, a été citée par Uvarov (1924, 1942) d'après un individu, en provenance de San Vincente, conservé au Muséum de Paris. Elle n'a jamais été retrouvée et paraît accidentelle dans l'Archipel.

Cyrtacanthacris tatarica tatarica (Linnaeus 1758).

1758. Gryllus (Locusta) tataricus Linnaeus, Syst. Nat., 10e édit., I, p. 432.

Cette sous-espèce est distribuée dans toule l'Afrique, essentiellement l'Afrique centrale, l'Afrique du Sud et Madagascar. Elle a été signalée une seule fois de l'Archipel par Bolivar (1889). Si la signalisation est exacte, cette espèce ne peut qu'être exceptionnelle dans les Iles du Cap-Vert.

\section{Eyprepocnemidinae}

Heteracris littoralis (Rambur 1838).

1838. Gryllus littoralis Rambur, Faun. Andal., p. 78, pl. 7, fig. 1, 2.

Cette espèce est largement distribuée dans toute la zone méditerranéenne ainsi que dans la zone soudano-sahélienne au sud du Sahara. Elle a été signalée des Iles du Cap-Vert par Uvarov (1921) et par Chopard (1958). Nous l'avons retrouvée dans toutes les îles visitées, à l'exception de Fogo.

Dans l'Archipel, $H$. littoralis paraît assez strictement inféodé à des milieux sableux (lits d'oueds...) colonisés par des espèces végétales du genre Suaeda Forsk. formant des buissons bas et denses. Bien qu'assez localisé, il peut être abondant et si la densité moyenne d'adultes sur l'Archipel ne dépasse pas 30 à l'hectare, nous avons pu observer jusqu'à plus de 1500 imagos par hectare sur San Vicente. Sur cette île, $H$. littoralis est d'ailleurs à la fois abondant (densité moyenne : 288 imagos/hect.) et fréquent puisqu'il est présent dans $68 \%$ des relevés et constitue l'une des 5 espèces les plus couramment rencontrées. Sa présence sur Fogo est possible car lcs milicux propices existent, surtout en zone côtière, mais ils n'ont pu être prospectés convenablement à cause des difficultés d'accès d'une part, du manque de temps d'autre part.

Eyprepocprifas insularis Donskoff 1983.

1983. Eyprepocprifas insularis Donskoff, Bull. Soc. ent. France, 87, année 1982, p. 346.

Cette espèce nouvelle, la seule espèce brachyptère de l'Archipel (fig. 1), vient d'être décrite par notre collègue et ami M. Donskoff, du Muséum de Paris, qui n'a pu la rapporter à aucun genre connu et a créé pour elle le genre Eyprepocprifas. Il nous est agréable de le remercier ici pour cet hommage rendu au Programme de Recherches interdisciplinaire français sur les Acridiens du Sahel (PRIFAS) ainsi que pour les nombreux services qu'il n'a cessé de nous prodiguer depuis de nombreuses années dans le domaine de la taxonomie des Acridiens d'Afrique.

Un seul exemplaire mâle de cette espèce a été capturé à une altitude d'environ $800 \mathrm{~m}$, sur l'île de San Nicolau, le long de la route de Tarrafal, au niveau d'un col 
situé au pied du Monte Gordo, point culminant de l'île avec $1340 \mathrm{~m}$. Cet Acridien a été aperçu sur un muret bordant la route. La zone environnante était rocheuse, la pente forte, l'ambiance humide, cette partie de l'île étant fréquemment dans les nuages. L'environnement général du lieu de capture est très hétérogène, constitué d'une mosaïque de sol nu, de végétation herbeuse et buissonnante (Furcraea gigantea Vent. en particulier) et d'affleurements rocheux.

\section{Calliptaminae}

Acorypha clara (Walker 1870).

1870. Caloptemus clarus Walker, Cat. Derm. Salt. Brit. Mus., 4e partie, p. 711.

L'aire de distribution de $A$. clara s'étend du Cap-Vert à l'Ethiopie à travers toute la zone soudano-sahélienne au Sud du Sahara. Cette espèce a été signalée à deux reprises de l'Archipel du Cap-Vert par Chopard $(1936,1958 b)$ de toutes les îles principales à l'exception de San Antao et de Brava.

Nous n'avons pu la retrouver que sur deux îles: tout d'abord en 1979, avec quelques larves sur 2 stations de l'île de Santiago (180 et 20 larves/hect.), puis en 1981 sur 3 stations de l'île de Fogo (respectivement: 420 imagos et 5310 larves/ hect.; 100 larves/hect.; 50 larves/hect.). Les populations observées sont pour l'essentiel en fin de développement larvaire, avec de nombreuses larves de dernier stade et quelques jeunes imagos. Le cycle de $A$. clara est probablement identique à celui observé sur le continent, à savoir univoltin avec diapause imaginale de saison sèche (Lecoq 1978).

\section{Acridinae}

Acrida turrila (Linnaeus 1758).

syn. Acrida carinulata (Bolivar 1889).

1758. Gryllus Acrida turritus Linnaeus, Syst. Nat., 10c édit., I, p. 427.

Cette espèce a d'abord été signalée et décrite des Iles du Cap-Vert sous le nom de $A$. carinulata et mise en synonymie par la suite avec $A$. turrita, espèce très largement distribuée à travers toute l'Afrique. Elle n'a pas été retrouvée depuis la signalisation de Bolivar.

\section{Oedipodinae}

Pseudosphingonotus savignyi (Saussure 1884).

1884. Sphingonotus savignyi Saussure, Mém. Soc. Phys. Genève, vol. XXVIII, fasc. 9, p. 198 et 208 (Savigny, Desc. Egypt., pl. 7, fig. 13).

Cette espèce occupe la plus grande partie de l'Afrique au Nord de l'équateur ainsi que le Sud-Ouest de l'Asie. Nous l'avons signalée pour la première fois de l'Archipel dès notre première mission en 1979 (Duranton et al. 1980). Harz (1982) la signale de Santiago. Pour notre part, nous l'avons observée sur cette île en 1979, ainsi que sur Boa Vista, San Nicolau, Maio et Fogo en 1980 et 1981. Cette espèce est à la fois fréquente (présente globalement dans $28 \%$ des relevés) et abondante (densité moyenne: 277 imagos par hectare). Sur Boa Vista, en 1980, P. savignyi constituait l'espèce la plus fréquente de l'île (présente dans $92 \%$ des relevés) ; une 
pullulation importante y a été observée, en zone côtière sablonneuse, avec des densités de près de 40000 imagos et 10000 larves par hectare.

Il peut paraître surprenant que cette espèce n'ait pas ćté signalée antéricurement, par Chopard en particulier, puisque $P$. savignyi est dans l'Archipel un Acridien commun et de grande taille (certes difficile à capturer car très actif) mais dont les mâles produisent de fortes stridulations tout à fait caractéristiques (Uvarov 1966). Il pourrait s'agir là d'un exemple d'introduction récente. Cette espèce n'aurait pas encore atteint dans les îles de l'Archipel son plein équilibre dans la mesure où elle se trouve encore absente de certaines îles présentant pourtant des caractéristiques écologiques apparemment favorables. Elle serait en voie d'expansion dans l'Archipel et mériterait d'ĉtre suivie de près dans les années à venir, d'autant qu'elle est un ravageur déclaré des jeunes plants forcsticrs depuis quel. ques années.

\section{Sphingonotus canariensis canariensis (Saussure 1884).}

1884. Sphingonotus savignyi stirps canariensis Saussure, Mém. Soc. Phys. Nat. Genève, vol. XXVIII, fasc. 9, p. 208.

S. c. canariensis est une sous-espèce présente dans une grande partie de l'Afrique au Nord de l'équateur ainsi que dans une partie du Proche-Orient. Elle a été décrite de l'Archipel du Cap-Vert par Saussure (1884) et a depuis été signalée de toutes les îles (Burr 1927, Mistshenko 1936, Chopard 19361958 b, Harz 1982). Nous l'avons retrouvée dans toutes les îles prospectées. Cette espèce est fréquente et moyennement abondante. On la trouve, selon les îles, dans 13 à $18 \%$ des relevés, à l'exception de Santiago où elle est observée dans $62 \%$ des cas et où une densité de 5790 imagos par hectare a même été notée. Des larves ont aussi été capturées sur toutes les îles (sauf sur Maio). Sur le continent, cette espèce possède une diapause imaginale de saison sèche et unc seule génération annuelle (Lecoq 1980).

Sphingonotus rubescens rubescens (Walker 1870).

1870. Oedipoda rubescens Walker, Zoologist, vol. V, fasc. 2, p. 2301.

$S$. $r$. rubescens possède une aire de répartition plus septentrionale que $S$. $c$. canariensis. On le rencontre dans une grande partie de l'Afrique au Nord du $13^{\circ}$ parallèle (elle semble limitée vers le Sud aux zones les plus arides au Sud du Sahara), le Sud de l'Europe et le Prochc-Orient jusqu'à l'Inde. Déjà signalé par Chopard $(1958 b)$, nous l'avons observé dans toutes les îles visitées. Il s'agit d'une espèce très abondante (densité moyenne: 522 imagos/hect.) et d'une des 4 ou 5 espèces les plus fréquentes puisqu'elle est présente dans $73 \%$ des relevés à l'état adulte. Il s'agit même de l'espèce la plus fréquente sur San Vicente et San Nicolau. Les larves sont également fréquentes et abondantes sur toutes les îles.

A noter que Harz (1982) ne signale que S. rubescens burri. En réalité - ainsi que nous avons pu le constater grâce à l'obligeance de $M$. W. Lobin qui nous a communiqué une partie du matéricl concerné - les récoltes de MM. Bauer, Friebe et Groh contenaient en mélange $S$. rubescens rubescens et $S$. rubescens burri.

\section{Sphingonotus rubescens burri Chopard 1936.} p. 34 .

1936. Sphingonotus rubescens Walk. ssp. burri Chopard. Rev. fr. Ent., vol. III, fasc. 1,

S. rubescens burri, décrit des Iles du Cap-Vert par Chopard en 1936, diffère principalement de la sous-espèce nominative par une taille beaucoup plus réduite 
et fut d'abord considéré comme une sous-espèce insulaire. En 1958, Chopard signale cependant que la forme burri semble se trouver partout avec le type et que l'hypothèse précédente ne saurait être retenue.

Nous avons effectivement retrouvé cette sous-espèce sur San Antao, San Nicolau et Boa Vista, à chaque fois en mélange avec le type et dans environ $1 \%$ des relevés (tabl. V).

Tablfau V. Densité à l'hectare des imagos de S. rubescens burri et de S. r. rubescens dans les relevés où la forme burri a été observée.

\begin{tabular}{lccc}
\multicolumn{1}{c}{ Ile } & Relevé $\mathrm{n}^{\circ}$ & S. r. burri & S. r. rubescens \\
San Antao & 515 & 170 & 440 \\
& 489 & 120 & 50 \\
Boa Vista & 266 & 200 & 290 \\
San Nicolau & 216 & 150 & 75 \\
& 231 & 68 & 373 \\
& 223 & 33 & 67 \\
& 240 & 21 & 3493 \\
& 233 & 25 & 98
\end{tabular}

Nous avons donc là une forme rare dont le statut de sous-espèce paraît devoir être remis en question. Il s'agirait soit d'une espèce particulière, soit d'une simple variation de taille apparaissant rarement au sein de l'espèce type.

Burr (1927) a observé en 1913 des Sphingonotus rubescens sur l'île de San Vicente. Cette signalisation a été rattachée par Chopard (1936) à $S$. r. burri car à l'époque $S$. $r$. rubescens n'était pas connue des Iles. Il apparaît maintenant que cette signalisation a beaucoup plus de chances de concerner $S$. $r$. rubescens bien plus abondant dans l'Archipel. Par ailleurs, Burr n'a jamais fait mention d'une réduction de taille des individus qu'il a pu observer ( «... an apparently local form of $S$. rubescens, Wlk... »).

Aiolopus thalassinus (Fabricius 1781).

1781. Gryllus thalassinus Fabricius, Spec. Ins., vol. I, p. 367.

A. thalassinus possède une très large aire de distribution : presque toute l'Afrique, le Sud de l'Europe et l'Asie du Sud-Ouest. L'espèce est signalée dans l'Archipel de toutes les îles principales à l'exception de Sal et Maio (Bolivar 1889, Chopard $19361958 b$ et Harz 1982). Nous avons pu la retrouver sur San Antao, San Vicente, Santiago et Fogo. Elle est peu fréquente (11\% des relevés pour les imagos) et peu abondante (32 imagos/hect. en moyenne) bien que les densités puissent être localement élevées; plus de 2000 imagos/hect. ont été observés à 3 reprises. Les larves sont notées à des densités voisines de celles des adultes avec un maximum de 7250 /hect. sur San Vicente.

Le cycle de cette espèce sur l'Archipel reste inconnu. En Afrique de l'Ouest, en zone soudanienne, elle présente 3 générations par an et une courte diapause imaginale en saison sèche (Lecoq 1978).

Aiolopus strepens (Latreille 1804).

1804. Acrydium strepens Latreille, Hist. nat. Crust. Ins., vol. XII, p. 14.

Cette espèce possède une distribution méditerranéenne typique (Hollis 1968). Elle a été signalée des Iles du Cap-Vert par Chopard (1936) et Harz (1958). Cepen- 
dant, nous ne l'avons pas retrouvée et tous les excmplaires capturés au cours de nos 3 missions correspondent indéniablement à l'espèce voisine $A$. simulator qui est d'ailleurs présente sur le continent africain à des latitudes voisines de celles de l'Archipel (ces déterminations ont été confirmées par M. Donskoff, du Muséum de Paris). Nous n'avons pu revoir les déterminations de Chopard, aucun exemplaire du matériel récolté par Lindberg et Panelius ne subsistant dans les collections du Musćum de Paris. Par ailleurs, Hollis (1968) dans sa révision du genre Aiolopus ne signale nullement les exemplaires en provenance des Iles du Cap-Vert. Par contre, il nous a été possible, grâce à l'obligeance de MM. Lobin et Schröder du Forschunginstitut Senckenberg de Francfort sur le Main, de vérifier les échantillons déterminés par Harz et de constater qu'il s'agissait incontestablement, à notre avis, de $A$. simulator et non de $A$. strepens.

Aiolopus simulator simulator (Walker 1870).

1870. Epacromia simulatrix Walker, Cat. Derm. Salt. Brit. Mus., 40 partie, p. 773.

A. s. simulator est une sous-espèce présente dans toute la zone soudano-sahélienne d'Afrique. Son aire de distribution s'étend vers l'Est jusqu'à l'Inde et, en Afrique de l'Est, s'incurve vers la région des grands lacs (Hollis 1968). Cette sousespèce n'a pas encore été signalée des Iles du Cap-Vert. Peut-être a-t-elle été confondue avec l'espèce précédente, $A$. strepens, dont la distribution est méditerranéenne (cf. supra).

A. s. simulator est unc sous-espèce fréquente, dont les imagos ont été trouvés dans $31 \%$ des relevés. Moyennement abondante (166 imagos/hect.), elle est observée sur toutes les îles hautes et est absente de Boa Vista et Maio (si ce n'est une larve de $3^{\text {e }}$ stade trouvéc sur Maio). Cette espèce est particulièrement fréquente sur Santiago ( $78 \%$ des cas) et dans une moindre mesure sur San Nicolau. Elle peut être localement très abondante (jusqu'à 6990 imagos par hectare sur Santiago). Les larves sont relativement nombreuses à l'époque des prospections. Sur le continent, $A$. s. simulator possède 2 générations annuelles et une diapause imaginale de saison sìche (Joyce 1952, Lecoq 1980).

Epacromius tergestinus (Charpentier 1825).

1825. Gryllus tergestinus Charpentier, Hor. ent., p. 139.

Cette espèce a été signalée une seule fois par Chopard (1958) qui considère sa présence aux Iles du Cap-Vert comme surprenante puisqu'il s'agit d'une espèce palćarctique, rare en Europe méridionale, localisće principalement en Europe centrale et orientale ainsi qu'en Asie centrale et en Chine (Saraiva 1961). Elle ne semble pas exister en Afrique à l'exception de deux signalisations en Egypte (Johnston 1956).

Chopard signale que la seule femelle capturée dans l'Archipel ressemble à un Insecte âgé ayant beaucoup volé (extrémités des ailes et des élytres fortement déchirées). Il ne peut s'agir que d'une espèce accidentelle, classée d'ailleurs par Johnston (1968) parmi les signalisations douteuses pour l'Afrique.

Trilophidia conturbata (Walker 1870).

1870. Epacromia conturbata Walker, Cat. Derm. Salt. Brit. Mus., 4e partie, p. 772.

Cette espèce a une distribution typiquement éthiopienne, occupant toute l'Afrique au Sud du Sahara. Signalce par Chopard (1958) et Harz (1982) de Maio, San- 
tiago, Fogo et Brava, nous l'avons retrouvée sur ces 3 premières îles. $T$. conturbata est une espèce au tempérament hygrophile, peu fréquente et peu abondante (47 imagos/hect. en moyenne) même si localement une densité d'adultes de $2960 /$ hect. a pu être dénombrée. Sur le continent, cette espèce possède une reproduction continue et 3 générations annuelles (Chapman 1962, Lecoq 1980).

\section{Acrotylus longipes (Charpentier 1843).}

1843. Oedipoda longipes Charpentier, Orth. disc. dep., pl. 54.

A. longipes se trouve dans toute l'Afrique au Nord de l'équateur ainsi que dans le Sud de l'Europe et l'Asie du Sud-Ouest. Cette espèce a été signalće dans les Iles du Cap-Vert à de nombreuses reprises par Butler (1881), Burr (1927), Chopard $(1936,1958 b)$, Harz (1982). Nous l'avons retrouvée dans toutes les îles prospectées. Il s'agit d'une des espèces les plus fréquentes et les plus abondantes des Iles (présente dans $61 \%$ des relevés; 495 imagos/hect.). Sur San Vicente, San Nicolau, Santiago et Fogo, elle se trouve dans plus de $75 \%$ des relevés. Des pullulations locales ont été notées avec une densité maximale de 13800 imagos par hectare sur Santiago où l'espèce est par ailleurs présente dans $81 \%$ des relevés. De tempérament plutôt xérophile, elle présente, au Soudan, une diapause imaginale de saison sèche (Joyce 1952).

Acrotylus patruelis (Herrich-Schäffer 1838).

1838. Gryllus patruelis Herrich-Schäffer, Faun. Ins. Germ., fasc. 157, pl. 18.

A. patruelis est également largement distribué dans toute l'Afrique, le Sud de l'Europe et l'Asie du Sud-Ouest. Il a été signalé par Chopard (1958 b) de toutes les îles à l'exclusion de Sal. Il s'agit donc d'une cspèce fréquente (présente dans $28 \%$ des relevés) que nous avons retrouvée sur toutes les îles, principalement sur Santiago; elle est assez rare sur Boa Vista et, d'une manière génćrale, peu abondante (96 imagos/hect. en moyenne).

Considéré d'une façon surprenante comme xérophile par Saraiva (1961), $A$. patruelis se trouve en fait soit dans les zones d'altitude, soit dans les fonds de "ribeiras" (sortes de cañons) et les endroits humides des zones basses. L'espèce présente donc un tempérament plutôt hygrophile.

Signalons que Chopard (1936), à la suite de Burr (1927), avait envisagé l'existence aux Iles du Cap-Vert d'une espèce voisine, méditerranćenne, A. insubricus, laquelle est d'ailleurs mentionnée de l'Archipel en 1954, par Chopard, dans sa liste des Orthoptères de la Macaronésie (signalisation reprise dans le catalogue de Johnston, en 1968). Cette hypothèse s'est, par la suite, trouvće infirmée par les faits et, dès 1958, Chopard ne signalait plus de l'Archipel que A. patruelis.

Oedaleus senegalensis (Krauss 1877).

1877. Pachutylus senegalensis Krauss, S.B. Akad. Wiss. Wien, vol. LXXVI, fasc. 1, p. 56, pl. 1, figs $9,9 \mathrm{~A}$.

$O$. senegalensis se rencontre dans toute l'Afrique au Nord de l'équateur ainsi que dans le Sud-Ouest de l'Asie (Ritchie 1981). C'est l'une des espèces les plus communes de l'Afrique occidentale en saison des pluies.

Il a été signalć de l'Archipel du Cap-Vert dès 1881 par Butler, puis par Bolivar 
(1889) et par tous les auteurs ultérieurs ayant travaillé sur les Acridiens de cette région. Il s'agit de l'espèce la plus fréquente et la plus abondante de l'Archipel; présente dans $79 \%$ des cas à l'état adulte, sa densité moyenne sur les îles est de 944 imagos par hectare. Sur Boa Vista, cette espèce est présente dans $97 \%$ des relevés; plusieurs pullulations ont été observées avec une densité maximale de plus de 22000 adultes par hectare. A l'époque des relevés, en fin de saison des pluies, les larves sont beaucoup moins abondantes que les adultes.

Cette espèce est, du point de vue économique, la plus importante de l'Archipel du Cap-Vert. Elle est responsable, chaque année, de dégâts notables aux cultures. particulièrement dans les îles basses, Boa Vista et Maio. Le cycle de $O$. senegalensis a été étudié sur l'Archipel par Saraiva (1962). Les œufs passent la saison sèche en diapause et il y aurait jusqu'à 2 générations par an en fonction de la durće de la saison des pluies, contre 3 générations possibles sur le continent où le cycle de cette espèce est maintenant bien connu (Batten 1969, Launois 1978, Lecoq 1978).

Oedaleus senegalensis var. dimidiatus Bolivar, 1889.

1889. Oedaleus senegalensis Krauss var. dimidiatus Bolivar, J. Sci. Acad. Lisboa, vol. I, fasc. 2, p. 105.

Cette variété, décrite en 1889 par Bolivar sur des exemplaires capturés aux Iles du Cap-Vert par Ferreira Borges, diffère de la forme typique par une coloration générale du corps beaucoup plus sombre. Les élytres, en particulier, sont de teinte sombre uniforme et le croissant noir de l'aile s'étend sur toute la partie apicale de celle-ci.

Nous avons toujours observé cette variété en mélange avec le type et en faible quantité. Elle est présente - dans $4 \%$ des relevés seulement - sur toutes les îles visitées à l'exclusion de San Vicente où elle n'a pas été capturće.

Cette variété est considérée par Ritchie $(1978,1981)$ comme une forme mélanique, faute d'avoir pu trouver des différences significatives (ou très faibles) entre les deux formes, tant au niveau de la morphologie génćrale que du complexe phallique. Concernant l'origine de cette forme mélanique, Ritchie envisage 3 hypothèses: l'influence des brûlis, celle du groupement ou un déterminisme génétique. A la suite d'observations personnelles, cette dernière hypothèse nous semblc devoir être retenue. En effet, nous avons pu, lors de notre dernier séjour aux Iles du CapVert en 1981, collecter une souche de huit adultes de $O$. senegalensis forme dimidiatus et la mettre en élevage au Centre de Recherches GERDAT de Montpellier. Quelques pontes furent obtenues au mois de novembre 1981 et des éclosions eurent lieu à partir de la mi-avril 1982 (soit 5 mois après les pontes). Les premiers adultes furent observés à la fin du mois de mai. Sur 23 individus obtenus à ce jour, 17 avaient l'aspect de la forme typique et 6 développaient le mélanisme caractéristique de la forme dimidiatus. L'existence d'un polymorphisme dû à une mutation récessive paraît donc plausible, d'autant que les brûlis sont inexistants dans les îles et que la forme dimidiatus a le plus souvent été capturéc au sein de populations de très faible densité. Cette forme mélanique a dû se développer à la suite d'une mutation survenue au niveau de la population insulaire; elle s'est ensuite trouvée limitée à l'Archipel, la direction des vents dominants rendant difficile la colonisation du continent par cette forme. Des migrations en sens inverse sont par contre tout à fait possibles et probables (Ritchie 1978). 
Locusta migratoria (Linnaeus 1758).

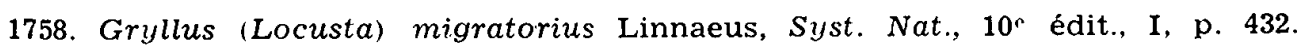

Le Criquet migrateur - dont l'aire de distribution est extrêmement vaste puisqu'elle couvre, outre l'Afrique, une bonne partic de l'Europe, de l'Asie et de l'Australie - a été signalé de l'Archipel du Cap-Vert à plusieurs reprises (Chopard 1936 1958, Harz 1982). Tous les individus observés appartenaient à la phase solitaire. Chopard (l.c.) note simplement une taille légèrement plus faible que celle des individus du continent africain. Nous avons pu retrouver cette espèce sur San Antao et Fogo. Elle est peu fréquente, peu abondante; la densité maximale observée a été de 720 imagos par hectare sur Fogo. Des larves ont également été notées en densité comparable à celle des imagos.

Le Criquet migrateur, de tempérament méso-hygrophile, se trouve, dans l'Archipel, localisé aux biotopes relativement hygrotrophes. Nous l'avons trouvé principalement dans les champs de canne à sucre irrigućs et les lits d'oueds sur San Antao, et dans les champs de maïs de moyenne altitude (400-1000 m) sur Fogo. Sur cette île un individu a été aperçu près du sommet du volcan de la Caldeira à 2620 m d'altitude.

Selon les régions du globe, plusieurs sous-espèces du Criquet migrateur sont distinguées. En fait, elles sont indiscernables par la morphométrie (Farrow \& Cólless 1980) et seules les caractéristiques bio-écologiques (sensibilité à la photopériode...) permettent de les séparer (Verdier 1967). On distingue, d'une part, les sous-espèces de la région paléarctique, univoltines, avec diapause embryonnaire pendant la saison fraîche, et, d'autre part, les sous-espèces des régions tropicales et sub-tropicales, plurivoltines, sans diapause à aucun stade de développement. Ces deux groupes sont, schématiquement, sćparés par la ceinture de zones désertiques de l'hémisphère Nord. Aux Iles du Cap-Vert, Saraiva (1961) considère que la sousespèce migratorioides n'existe pas. En fait, les différents auteurs ayant signalé le Criquet migrateur dans l'Archipel n'ont jamais fait mention d'une sous-espèce particulière. La sous-espèce en question est vraisemblablement L. migratoria migratorioides que l'on trouve sur le continent proche à une latitude identique, ainsi que dans toute l'Afrique au Sud du Sahara, et qui peut très certainement parvenir du continent aux îles grâce aux vents d'harmattan. Une étude biologique précise permettrait éventuellement de confirmer le statut de cette sous-espèce.

\section{Truxalinae}

Truxalis nasuta (Linnaeus 1758).

1758. Gryllus Acrida nasutus Linnaeus, Syst. Nat., $10^{\circ}$ édit., I, p. 427.

Cette espèce a été signalće par Bolivar, en 1889, et n'a jamais ćtć rctrouvée depuis. Il s'agit d'un Acridien de grande taille, très caractéristique, qui était considéré autrefois comme très largement répandu, en particulier dans toute l'Afrique. Dirsh (1965), limite son aire de distribution à l'Asie du Sud-Ouest et à la région méditerranćenne. Il s'agit à l'évidence d'une espèce tout à fait accidentelle pour l'Archipel. Elle paraît plus fréquente aux Canaries (Chopard 1954, Johnsen 1974). 


\section{Gomphocerinae}

Leva bonneti (Bolivar 1885).

syn. Stenohippus bonneti.

1885. Stenobothrus bonneti Bolivar, Le Naturaliste, vol. VII, p. 116.

L. bonneti est distribué dans toute l'Afrique occidentale dans les zones de savanes. L'espèce a été signalée des Iles du Cap-Vert par Chopard $(1936,1958 b)$ et par Harz (1982) de Santiago et Fogo. Nous l'avons retrouvée également sur San Antao, San Nicolau et Maio. Elle est peu fréquente (17\% des relevés) mais peut être abondante localement. Des densités de 1070 imagos et 5280 larves par hectare ont été notées sur San Antao. Des larves de tous stades ont été capturées, essentiellement des jeunes avant retournement des ébauches alaires. Quelques éclosions ont même été observées. On est donc, lors des prospections, en plein développement larvaire, les adultes de cette génération devant apparaître en majorité un peu plus tardivement.

Leva epacromioides (Krauss 1877).

syn. Stenohippus epacromioides.

1877. Stenobothrus epacromioides Krauss, S.B. Akad. Wiss. Wien, vol. LXXVI, fasc. 1, p. 54.

Cette espèce est voisine de la précédente avec laquelle elle est d'ailleurs mise en synonymie dans le catalogue de Johnston (1968). Elle s'en distingue cependant par les carènes latérales du pronotum parallèles dans la prozone chez bonneti et incurvées chez epacromioides (Donskoff 1975 comm. pers., Lecoq 1979). En attendant une révision du genre (en cours par N. Jago) nous conserverons son identité à cette espece. Aux Iles du Cap-Vert, nous l'avons très généralement trouvée associée à l'espèce précédente.

L. epacromioides n'avait pas encore été signalé de l'Archipel, même aux époques où la synonymie avec bonneti n'avait pas encore été établie. Nous avons trouvé les deux espèces dans les mêmes îles et les populations ont les mêmes caractéristiques : fréquence et abondance faibles (13\% des relevés ; 14 imagos/hect. en moyenne), quelques "pullulations " locales (jusqu'à 1210 larves et 7440 imagos par hectare), un développement larvaire en cours. En conclusion, des caractéristiques biologiques et écologiques similaires, qui restent encore à préciser d'une manière plus fine, mais qui pourraient inciter à penser que bonneti et epacromioides sont effectivement synonymes.

\section{Ochrilidia geniculata (Bolivar 1913).}

1913. Platypterna geniculata Bolivar, Novit. Zool., vol. XX, p. 607.

O. geniculata est répandu dans une grande partie de l'Afrique au Nord du $10^{*}$ parallèle ainsi qu'en $\Lambda$ sie du Sud-Ouest jusqu'à l'Inde (Jago 1977). L'espèce a été signalée des Iles du Cap-Vert une seule fois par Chopard (1958) sur Boa Vista. Nous l'avons retrouvée également sur Maio. Il s'agit d'une espèce rare, présente dans 4 relevés seulement, dans les touffes de Graminées des milieux sableux dunaires. Les densités sont cependant notables (un maximum de 450 imagos et 1360 lar- 
ves par hectare observé sur Maio). Dans l'ensemble, le développement larvaire est en cours et la majorité des adultes doit apparaître plus tardivement.

Brachycrotaphus tryxalicerus (Fischer 1853).

1853. Opomala tryxalicera Fischer Orth. Eur., p. 305, pl. 15, fig. 8.

$B$. tryxalicerus est répandu dans toute l'Afrique des savanes et le Sud de l'Europe. Un mâle a été signalé par Chopard (1958) de Santiago (Pico da Antonio). Cette espèce, relativement fréquente sur le continent, n'a pas été retrouvée dans l'Archipel et doit être considérée comme rare ou d'introduction accidentelle en provenance d'Afrique.

\section{Espèce non déterminée:}

Nous avons capturé dans le cratère de Cova, sur San Antao, des larves qui n'appartenaient à aucune des espèces précédentes. En l'absence d'adultes, il nous a été impossible, pour le moment, de tenter une détermination (fig. 2).

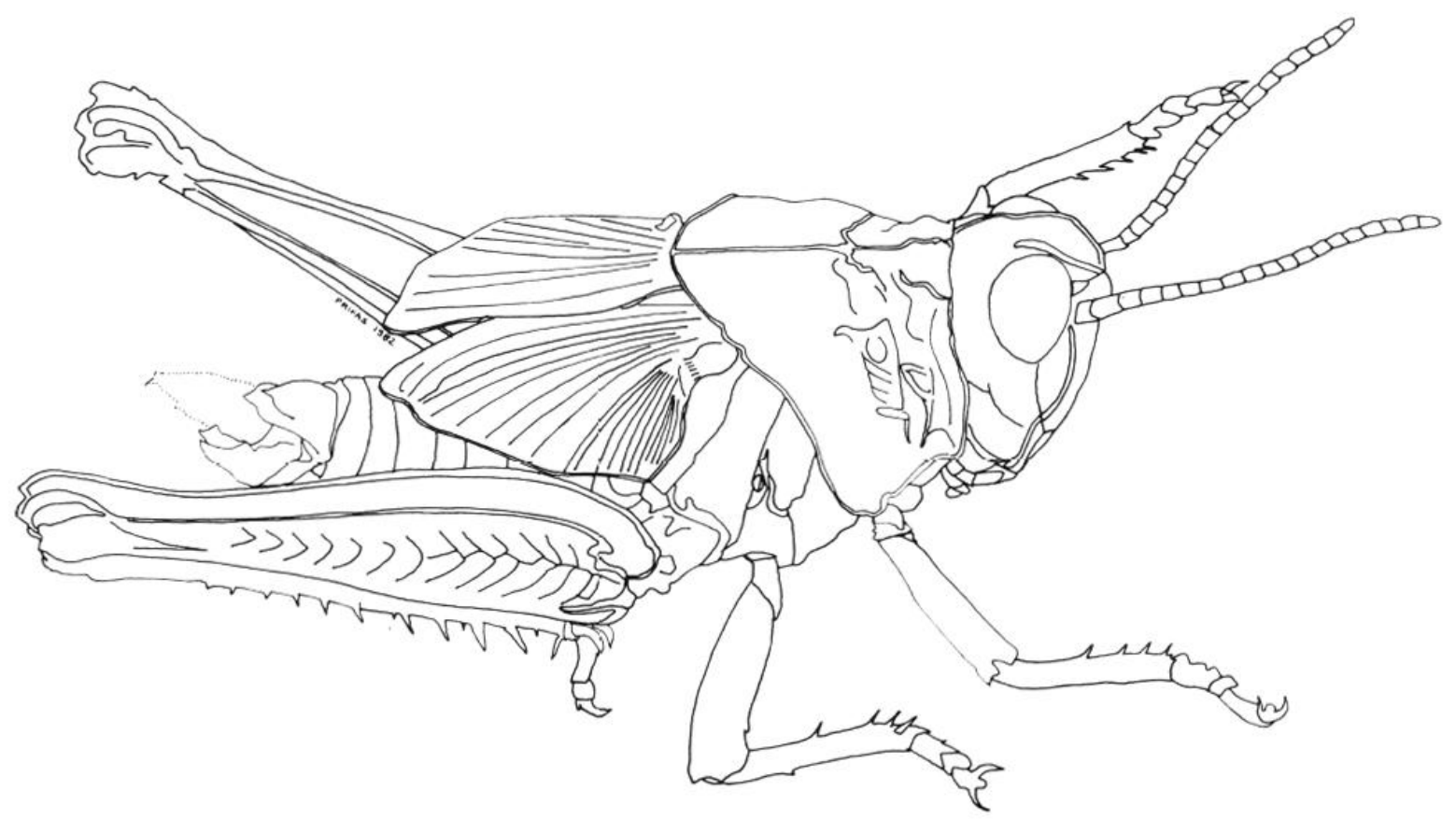

Fig. 2, larve de dernier stade d'une espèce indéterminée capturée sur l'île de San Antao le 12 octobre 1981 dans le cratère de Cova.

\section{Corrections :}

Sphingonotus azurescens Rambur, 1838, signalé de Fogo par Chopard (1936) et cité par Johnston (1956) et Harz (1982) doit être supprimé de la liste des Acridiens des Iles du Cap-Vert en accord avec Chopard $(1954,1958)$ et Johnston (1968).

REMERCIEMENTS: Les auteurs remercient tout particulièrement les autorités Cap-Verdiennes et la sous-Direction de la Recherche du Ministère français de la Coopération et du Développement grâce auxquelles les missions sur l'Archipel ont été possibles, M. André Duhart, Conseiller F.A.O., pour toutes les facilités qu'il nous a ménagées dans les différentes îles, et $M$. Michel Donskoff, entomologiste CNRS au Muséum de Paris, pour les identifications d'espèces. 


\section{REFERENCES}

BATtEN (A.), 1969. - The Senegalese grasshopper Oedaleus senegalensis Krauss (J. appl. Ecol., $6:$ 27-45).

Bolivar (I.), 1889. - Ortópteros de Africa del Museo de Lisboa (J. Sci. Acad. Lisboa, 1 (2) : 73-112, 150-173, 211-232, 1 pl.).

Burr (M.), 1927. - Notes from the Cape Verde Islands (Ent. Rec., 39: 91-95).

BUtLer (A.G.), 1881. - An account of the zoological collections made during the survey of H.M.S. Alert in the Straits of Magellan and on the coast of Patagonia (Proc. zool. Soc. Lond., 1881: 2-140).

Chapman (R.F.), 1962. - The ccology and distribution of grasshoppers in Ghana (Proc. zool. Soc. Lond., 139 (1) : 1-66).

Chevalier (A.), 1935. - Les Iles du Cap-Vert. Géographie, Biogéographie, Agriculture, Flore de l'Archipel (Rev. Bot. appl. Agric. trop., 15 (170-171) : 736-1090).

Chopard (L.), 1936. - Mission de M. A. Chevalier aux Iles du Cap-Vert (1934) (Rev. franc. Ent., 3: 88-96).

- 1946. - Les Orthoptéroïdes des îles atlantides (Mém. Soc. Biogéogr., 8 : 199-208).

- 1954. - Contributions entomologiques de l'expédition finlandaise aux Canarics 1947-1957. No 7. Insectes Orthoptéroïdes récoltés aux Iles Canaries par M. H. Lindberg (Commentat. biol. Helsingfors, 14 (7) : 1-15).

- 1958a. - La réserve naturelle intégrale du Mont Nimba. III. Acridiens (Mém. IFAN, $53: 127-153)$.

- 1958b. - Orthopteroidea. Résultats de l'expédition zoologique du Professeur Dr. Hakan Lindberg aux Iles du Cap-Vert durant l'hiver 1953-1954. No 16 (Commentat. biol. Helsingfors, 17 (3) : 1-17).

- 1958c. - Mission du Muséum dans les îles du Golfe de Guinćc. Entomologic, VI. Orthoptéroïdes (Bull. Soc. ent. France, 63: 73-85).

Davey (J.T.), Descamps (M.) \& Drmange (R.), 1959. - Notes on the Acrididae of the French Sudan with special reference to the Central Niger Delta (Bull. IFAN, 21 (A) : 60-112, 565-600).

Descamps (M.), 1965. - Acridoïdes du Mali (deuxième contribution). Régions de San et de Sikasso (zone soudanaise) (Bull. IFAN, 27 (A) : 922-962, 1259-1314).

- 1968. - Acridoïdes du Tchad (Bull. IFAN, 30 (A) : 535-588).

Dirsh (V.M.) \& Uvirov (B.P.), 1953. - Tree locusts of the genus Anacridizim (Orthoptera, Acrididae) (Eos (Madrid), 29 (1): 7-69).

- 1965. - The African Genera of Acridoidea. University Press (Cambridge), 579 p.

DonskofF (M.), 1983. - Un Acridien nouveau des Iles du Cap Vert: Eyprepocprifas insularis n. gen. et n. sp. (Orth. Acrididac) (Bull. Soc. ent. France, 87 (9-10), 1982 : 345-349).

Duranton (J.F.), Launois (M.), Lawois-Luong (M.H.) \& Lecoo (M.), 1980. - Les Acridiens dı Sahel aux Iles du Cap-Vert. Ministère de la Coopération et GERDAT (Paris), affiche polychrome $60 \times 90 \mathrm{~cm}$.

- 1982. - Manuel de prospection acridienne en zone tropicale sèche. Ministère des Relations Extérieures (Coopération et Développement) el GERDAT (Paris), T1 et T2: $1500 \mathrm{p}$.

FARRow (R.A.) \& Colufss (D.H.), 1980. - Analysis of interrelationships of geographical races of Locusta migratoria (Linnacus) (Orthoptera: Acrididae) by numerical laxonomy with special reference to subspeciation in tropics and affinities of the australian race (Acrida, 9: 77-99).

Golding (F.D.), 1948. - The Acrididac (Orthoptera) of Nigeria (Trans. R. ent. Soc. Lond., 99: 517-587).

HARZ (K.), 1982. - Zur Orthopteren Fauna (s. lat.) der Kapverdischen Inseln (Cour. Forsch.-Inst. Senckenberg, 52: 153-154).

Hollis (D.), 1970. - A revision of the genus Aiolopus Ficber (Orthoptera: Acridoidea) (Bull. Br. Mus. nat. Hist. (Ent.), 22 (7) : 309-355). 
HstCNg (C.C.) \& Kerax (D.K.McE.), 1975. - Preliminary observations on conica-bispinosacognata group of the genus Pyrgomorpha Audinet-Serville (Orthoptera: Pyrgomorphidae) (Acrida, $4: 57-68$ ).

JaGo (N.), 1977. - Revision of the genus Ochrilidia Stal, 1873, with comments on the genera Sporobolizis Uvarov, 1941 and Platypternodes I. Bolivar, 1908 (Orthoptera, Acrididae, Gomphocerinae) (Acrida, 6: 163-217).

Johssex (P.), 1970. - Notes on African Acridoidea in Danish Museums. Part I (Natura Jutlandica, 15 : 121-162).

- 1974. - Contribution to the knowledge of the Dermaptera, Orthoptera and Dictyoptera of the Canary Islands (Natura Jutlandica, 17: 25-57).

- 1977. - Description of the male Anacridium eximium (Sjöstedt, 1918) from Cape Verde Islands (Orthopteroidea: Catantopidae) (Vieraea, 6 (2): 227-230).

- 1981. - A dry-scason collection of short-horned Grasshoppers from Gambia (Orthoptera: Acridomorphoidea) (Ent. scand., 12: 81-98).

Johisston (H.B.), 1956. - Annotated Catalogue of African Grasshoppers. University Press (Cambridge), 834 p.

- 1968. - Annotated Catalogue of African Grasshoppers. Supplement. University Press (Cambridge), 448 p.

JoYcE: (R.J.V.), 1952. - The ecology of grasshoppers in cast central Sudan (Anti-locust Bull., 11 : 99 p.).

KaRsh (F.), 1888. - Beiträge zu Ignacio Bolivar's Monografía de los Pirgomorfinos (Madrid, 1884) (Ent. Nachr., $14:$ 328-335, 340-346, 355-361).

Lauxors (M.), 1972. - Contribution à l'étude du fonctionnement ovarien du Criquet migrateur Locusta migratoria capito Sauss. dans la nature (Ann. Zool.-Ecol. anim., $\mathrm{n}^{\circ}$ hors-série: 55-116).

- 1978. - Manuel pratique d'identification des principaux Acridiens du Sahel. Ministère de la Coopération et GERDAT (Paris): 304 p.

LFCO0 (M.), 1978. - Biologie et dynamique d'un peuplement acridien de zone soudanienne en Afrique de l'Ouest (Orthoptera, Acrididae) (Ann. Soc. ent. France (N.S.) 14 (4) : 603-681).

- 1979. - Clés de détermination des Acridiens des zones sahéliennes et soudaniennes en Afrique de l'Ouest (Bull. IFAN, série A, 41 (3): 531-595).

- 1980. - Biologie et dynamique d'un peuplement acridien de zone soudanienne en Afrique de l'Ouest (Orthoptera, Acrididae). Note complémentaire (Ann. Soc. ent. France (N.S.) 16 (1): 49-73).

Mistshenko (L.), 1936. - Revision of Palearctic species of the genus Sphingonotus Fieber (EOS (Madrid), 12 (1-2): 65-192).

PEDGLeY (D.), 1981. - Desert Locust forecasting manual. C.O.P.R. (London). T.1 + T.2: $270+144 \mathrm{p}$.

ReHn (J.A.G.), 1944. - South African bird locust records and notes (Orthoptera, Acrididae, Cyrtacanthacridinae, group Cyrtacanthacres) (Notul. nat. Acad. Philad., 137 : 11 p.).

Ritchis: (J.M.), 1978. - Melanism in Oedaleus senegalensis and other Ocdipodines (Orthoptera, Acrididae) (J. nat. Hist., 12 : 153-162).

- 1981. - A taxonomic revision of the genus Oedaleus Fieber (Orthoptera, Acrididae) (Bull. Br. Mus. nat. Hist. (Ent.), 42 (3): 83-183).

Roblot (M.), 1950. - Les Acridiens migrateurs au Soudan (Acad. Agric. Fr. (C.R.), 3 : 102-107).

Saraiva (A.C.), 1961. - Conspectus da entomofauna cabo-verdiana (Estud., Ensaios, Docum. Jta. Invest. Ultramar (Lisboa) : 83).

- 1962. - Plague locusts - Oedaleus senegalensis (Krauss) and Schistocerca gregaria (Forskal) in the Cape Verde Islands. Estud. agron., 3: 61-89.

Uvarov (B.P.), 1921. - Notes on Orthoptera in the British Museum. I. The group Euprepocnemini (Trans. ent. Soc. Lond., 1921 (1, 2): 106-144).

1924. - A revision of the Old World Cyrtacanthacrini. IV - V (Ann. Mag. nat. Hist., Ser. 9, $13: 1-19 ; 14: 96-113)$. 
- 1942. - A revision of the genus Ornithacris Uvarov, 1924 (Ann. Mag. nat. Hist., Ser. 11, 9: 135-140).

- 1966. - Grasshoppers and Locusts. A handbook of general acridology. University Press (Cambridge) : $481 \mathrm{p}$.

VERDIER (M.), 1967. - Deux contributions à l'effet du facteur photopériodique dans le développement du Criquet migrateur vers sa limite Nord-Ouest. Thèse de Doctorat d'Etat (Paris): 243 p. (public. multigr.).

Wintrebert (D.), 1955. - La ponte et l'éclosion du Criquet nomade (Nomadacris septemfasciata Serv.) dans la zone d'inondation du Niger (Soudan Français) (Agron. Trop., 10: 610-616).

(Groupement d'Etudes et de Recherches pour le Développement de l'Agronomie tropicale (GERDAT). Programme de Recherches interdisciplinaire français sur les Acridiens du Sahel, B.P. 5035, F-34032 Montpellier Cedex).

\author{
Contribution à la connaissance des Micro-Histeridae \\ des îles Fidji, Tonga, Samoa et Cook \\ [CoL.] \\ par Yves GomY
}

Dans la très importante collection de Micro-Histeridae envoyée par le Dr E.G. Matthews du South Australian Museum d'Adelaïde, se trouvait, en plus de l'abondant matériel australien proprement dit, un petit lot de 27 exemplaires des Iles Fidji capturés par A.M. Lea en 1924. C'est l'étude dc ces Insectes, auxquels j'ai ajouté 12 exemplaires provenant des récoltes du Dr G. Kuschel (Auckland) dans cet archipel en 1975 et 1977, et 3 exemplaires des Iles Tonga, 5 ex. des Samoa et 2 ex. des Iles Cook, qui fait l'objet de la présente étude. Celle-ci me permet de décrire cinq espèces nouvelles et de compléter la répartition géographique de plusieurs autres. Je remercie vivement une nouvelle fois mes collègues E.G. Matthews et G. Kuschel pour la confiance qu'ils me témoignent en m'envoyant ainsi du matériel rare de régions lointaines encore très mal connues des naturalistes en général et des entomologistes européens en particulier. Je remercie également le $\mathrm{Dr}$ $\mathrm{R}$. Poggi de Gênes qui m'a permis de revoir la série typique d'Acritus indignus Schmidt, et de désigner le lectotype.

\title{
ABRAEINAE ACRITINI
}

\section{Genre Acritus Le Conte (1853)}

Acritus fidjicus $\mathrm{n}$. sp. (fig. 1 a et e). Cette espèce fait penser à première vue aux deux espèces australiennes récemment décrites: $A$. dogueti Gomy et $A$. kuscheli Gomy (1983, sous presse) dont elle a la forme générale et la ligne de gros points à la base du pronotum. L'examen de la région pro-méso-métasternale permet de la distinguer immédiatement (fig. 1, a). 